JOURNAL OF THE

AMERICAN MATHEMATICAL SOCIETY

Volume 26, Number 4, October 2013, Pages 901-937

S 0894-0347(2013)00769-9

Article electronically published on March 27, 2013

\title{
HOMOGENIZATION OF ELLIPTIC SYSTEMS WITH NEUMANN BOUNDARY CONDITIONS
}

\author{
CARLOS E. KENIG, FANGHUA LIN, AND ZHONGWEI SHEN
}

\section{InTRODUCTION AND STATEMENT OF MAIN RESUlts}

The main purpose of this work is to study uniform regularity estimates for a family of elliptic operators $\left\{\mathcal{L}_{\varepsilon}, \varepsilon>0\right\}$, arising in the theory of homogenization, with rapidly oscillating periodic coefficients. We establish sharp $W^{1, p}$ estimates, Lipschitz estimates, and nontangential maximal function estimates, which are uniform in the parameter $\varepsilon$, on solutions with Neumann boundary conditions.

Specifically, we consider

$$
\mathcal{L}_{\varepsilon}=-\frac{\partial}{\partial x_{i}}\left[a_{i j}^{\alpha \beta}\left(\frac{x}{\varepsilon}\right) \frac{\partial}{\partial x_{j}}\right]=-\operatorname{div}\left[A\left(\frac{x}{\varepsilon}\right) \nabla\right],
$$

where $\varepsilon>0$. We assume that the coefficient matrix $A(y)=\left(a_{i j}^{\alpha \beta}(y)\right)$ with $1 \leq$ $i, j \leq d$ and $1 \leq \alpha, \beta \leq m$ is real and satisfies the ellipticity condition

$$
\mu|\xi|^{2} \leq a_{i j}^{\alpha \beta}(y) \xi_{i}^{\alpha} \xi_{j}^{\beta} \leq \frac{1}{\mu}|\xi|^{2} \quad \text { for } y \in \mathbb{R}^{d} \text { and } \xi=\left(\xi_{i}^{\alpha}\right) \in \mathbb{R}^{d m},
$$

where $\mu>0$, the periodicity condition

$$
A(y+z)=A(y) \quad \text { for } y \in \mathbb{R}^{d} \text { and } z \in \mathbb{Z}^{d},
$$

and the smoothness condition

$$
|A(x)-A(y)| \leq \tau|x-y|^{\lambda} \quad \text { for some } \lambda \in(0,1) \text { and } \tau \geq 0 .
$$

We will say $A \in \Lambda(\mu, \lambda, \tau)$ if $A=A(y)$ satisfies conditions (1.2), (1.3), and (1.4).

Let $f \in L^{2}(\Omega)$ and $g \in W^{-1 / 2,2}(\partial \Omega)$. Consider the Neumann boundary value problem

$$
\left\{\begin{aligned}
\mathcal{L}_{\varepsilon}\left(u_{\varepsilon}\right) & =\operatorname{div}(f) & & \text { in } \Omega, \\
\frac{\partial u_{\varepsilon}}{\partial \nu_{\varepsilon}} & =g-n \cdot f & & \text { on } \partial \Omega,
\end{aligned}\right.
$$

where

$$
\left(\frac{\partial u_{\varepsilon}}{\partial \nu_{\varepsilon}}\right)^{\alpha}=n_{i}(x) a_{i j}^{\alpha \beta}\left(\frac{x}{\varepsilon}\right) \frac{\partial u_{\varepsilon}^{\beta}}{\partial x_{j}}
$$

denotes the conormal derivative associated with $\mathcal{L}_{\varepsilon}$ and $n=\left(n_{1}, \ldots, n_{d}\right)$ is the outward unit normal to $\partial \Omega$. Assume that $\int_{\Omega} u_{\varepsilon}=0$. It is known from the theory of

Received by the editors October 28, 2010 and, in revised form, February 26, 2013.

2010 Mathematics Subject Classification. Primary 35J57.

The first author was supported in part by NSF grant DMS-0968472.

The second author was supported in part by NSF grant DMS-0700517.

The third author was supported in part by NSF grant DMS-0855294. 
homogenization that under the assumptions (1.2)-(1.3), $u_{\varepsilon} \rightarrow u_{0}$ weakly in $W^{1,2}(\Omega)$ as $\varepsilon \rightarrow 0$, where $\mathcal{L}_{0}\left(u_{0}\right)=\operatorname{div}(f)$ in $\Omega$ and $\frac{\partial u_{0}}{\partial \nu_{0}}=g-n \cdot f$ on $\partial \Omega$. Moreover, the homogenized operator $\mathcal{L}_{0}$ is an elliptic operator with constant coefficients satisfying (1.2) and depending only on the matrix $A$ (see e.g. 8]).

In this paper we shall be interested in sharp regularity estimates of $u_{\varepsilon}$, which are uniform in the parameter $\varepsilon$, assuming that the data are in $L^{p}$ or Besov or Hölder spaces. The following three theorems are the main results of the paper. Note that the symmetry condition $A^{*}=A$, i.e.,

$$
a_{i j}^{\alpha \beta}(y)=a_{j i}^{\beta \alpha}(y) \quad \text { for } 1 \leq i, j \leq d \text { and } 1 \leq \alpha, \beta \leq m,
$$

is also imposed in Theorems 1.2 and 1.3 .

Theorem 1.1 ( $W^{1, p}$ estimates). Suppose $A \in \Lambda(\mu, \lambda, \tau)$ and $1<p<\infty$. Let $\Omega$ be a bounded $C^{1, \alpha}$ domain for some $0<\alpha<1$. Let $g=\left(g^{\beta}\right) \in B^{-1 / p, p}(\partial \Omega)$, $f=\left(f_{j}^{\beta}\right) \in L^{p}(\Omega)$, and $F=\left(F^{\beta}\right) \in L^{q}(\Omega)$, where $q=\frac{p d}{p+d}$ for $p>\frac{d}{d-1}$ and $q>1$ for $1<p \leq \frac{d}{d-1}$. Then, if $F$ and $g$ satisfy the compatibility condition $\int_{\Omega} F^{\beta}+\left\langle g^{\beta}, 1\right\rangle=0$ for $1 \leq \beta \leq m$, the weak solutions to

$$
\left\{\begin{array}{rlrl}
\mathcal{L}_{\varepsilon}\left(u_{\varepsilon}\right) & =\operatorname{div}(f)+F & & \text { in } \Omega, \\
\frac{\partial u_{\varepsilon}}{\partial \nu_{\varepsilon}} & =g-n \cdot f & & \text { on } \partial \Omega, \\
u_{\varepsilon} & \in W^{1, p}(\Omega) &
\end{array}\right.
$$

satisfy the estimate

$$
\left\|\nabla u_{\varepsilon}\right\|_{L^{p}(\Omega)} \leq C\left\{\|f\|_{L^{p}(\Omega)}+\|F\|_{L^{q}(\Omega)}+\|g\|_{B^{-1 / p, p}(\partial \Omega)}\right\},
$$

where $C>0$ depends only on $d, m, p, q, \mu, \lambda, \tau$, and $\Omega$.

Theorem 1.2 (Lipschitz estimates). Suppose that $A \in \Lambda(\mu, \lambda, \tau)$ and $A^{*}=A$. Let $\Omega$ be a bounded $C^{1, \alpha}$ domain, let $0<\eta<\alpha<1$, and let $q>d$. Then, for any $g \in C^{\eta}(\partial \Omega)$ and $F \in L^{q}(\Omega)$ with $\int_{\Omega} F+\int_{\partial \Omega} g=0$, the weak solutions to

$$
\left\{\begin{array}{rlrl}
\mathcal{L}_{\varepsilon}\left(u_{\varepsilon}\right) & =F & & \text { in } \Omega, \\
\frac{\partial u_{\varepsilon}}{\partial \nu_{\varepsilon}} & =g & & \text { on } \partial \Omega, \\
\left|\nabla u_{\varepsilon}\right| & \in L^{\infty}(\Omega) &
\end{array}\right.
$$

satisfy the estimate

$$
\left\|\nabla u_{\varepsilon}\right\|_{L^{\infty}(\Omega)} \leq C\left\{\|g\|_{C^{\eta}(\partial \Omega)}+\|F\|_{L^{q}(\Omega)}\right\},
$$

where $C>0$ depends only on $d, m, \eta, q, \mu, \lambda, \tau$, and $\Omega$.

Theorem 1.3 (Nontangential maximal function estimates). Suppose that $A \in$ $\Lambda(\mu, \lambda, \tau)$ and $A=A^{*}$. Let $\Omega$ be a bounded $C^{1, \alpha}$ domain and let $1<p<\infty$. Then, for any $g \in L^{p}(\partial \Omega)$ with mean value zero, the weak solutions to

$$
\left\{\begin{array}{rlrl}
\mathcal{L}_{\varepsilon}\left(u_{\varepsilon}\right) & =0 & & \text { in } \Omega, \\
\frac{\partial u_{\varepsilon}}{\partial \nu_{\varepsilon}} & =g & & \text { on } \partial \Omega, \\
\left(\nabla u_{\varepsilon}\right)^{*} & \in L^{p}(\partial \Omega) &
\end{array}\right.
$$


satisfy the estimate

$$
\left\|\left(\nabla u_{\varepsilon}\right)^{*}\right\|_{L^{p}(\partial \Omega)}+\left\|\nabla u_{\varepsilon}\right\|_{L^{q}(\Omega)} \leq C\|g\|_{L^{p}(\partial \Omega)},
$$

where $q=\frac{p d}{d-1}$ and $C>0$ depends only on $d, m, p, \mu, \lambda, \tau$, and $\Omega$.

A few remarks on notation are in order. In Theorem 1.1, $B^{-1 / p, p}(\partial \Omega)$ is the dual of the Besov space $B^{1 / p, p^{\prime}}(\partial \Omega)$ on $\partial \Omega$, where $p^{\prime}=\frac{p}{p-1}$, and $\left\langle g^{\beta}, 1\right\rangle$ denotes the action of $g^{\beta}$ on the function 1. By a weak solution $u$ to (1.8), we mean that $u \in W^{1, p}(\Omega)$ and that it satisfies

$$
\int_{\Omega} a_{i j}^{\alpha \beta}\left(\frac{x}{\varepsilon}\right) \frac{\partial u_{\varepsilon}^{\beta}}{\partial x_{j}} \cdot \frac{\partial \varphi^{\alpha}}{\partial x_{i}} d x=\int_{\Omega}\left\{-f_{i}^{\alpha} \frac{\partial \varphi^{\alpha}}{\partial x_{i}}+F^{\alpha} \varphi^{\alpha}\right\} d x+\left\langle g^{\alpha}, \varphi^{\alpha}\right\rangle,
$$

for any $\varphi=\left(\varphi^{\alpha}\right) \in C_{0}^{1}\left(\mathbb{R}^{d}\right)$. In Theorem 1.3 we have used $\left(\nabla u_{\varepsilon}\right)^{*}$ to denote the nontangential maximal function of $\nabla u_{\varepsilon}$. We point out that the Lipschitz estimate in Theorem 1.2 is sharp. Even with $C^{\infty}$ data, one cannot expect higher-order uniform estimates of $u_{\varepsilon}$, as $\nabla u_{\varepsilon}$ is known to converge to $\nabla u_{0}$ only weakly. As a result, the use of nontangential maximal functions in Theorem 1.3 to describe the sharp regularity of solutions with $L^{p}$ Neumann data appears to be natural and necessary. Also note that under the conditions (1.2) and (1.4), the existence and uniqueness (modulo additive constants) of solutions to (1.8), (1.10), and (1.12) with sharp regularity estimates are more or less well known (see e.g. [1,2, 32, ). What is new here is that with the additional periodicity assumption (1.3), the constants $C$ in the regularity estimates (1.9), (1.11), and (1.13) are independent of $\varepsilon$.

In the case of the Dirichlet boundary condition $u_{\varepsilon}=g$ on $\partial \Omega$ with $g \in B^{1 / p^{\prime}, p}(\partial \Omega)$ or $g \in C^{1, \eta}(\partial \Omega)$, results analogous to Theorems 1.1 and 1.2 were established by Avellaneda and Lin in [3, 7] for $C^{1, \alpha}$ domains (without the assumption $A^{*}=A$ ). They also obtained the nontangential maximal function estimate $\left\|\left(u_{\varepsilon}\right)^{*}\right\|_{L^{p}(\partial \Omega)} \leq$ $C\|g\|_{L^{p}(\partial \Omega)}$ for solutions of $\mathcal{L}_{\varepsilon}\left(u_{\varepsilon}\right)=0$ in $\Omega$ (the case $m=1$ was given in [4]). As was noted in [3], uniform regularity estimates, in addition to being of independent interest, have applications to the homogenization of the boundary control of distributed systems [6, 26, 27]. Furthermore, they can be used to estimate convergence rates of $u_{\varepsilon} \rightarrow u_{0}$ as $\varepsilon \rightarrow 0$. In particular, it was proved in 3 that $\left\|u_{\varepsilon}-u_{0}\right\|_{L^{\infty}(\Omega)}=O(\varepsilon)$, if $\mathcal{L}_{\varepsilon}\left(u_{\varepsilon}\right)=\operatorname{div}(f)$ in $\Omega, u_{\varepsilon}=g$ on $\partial \Omega$, and $f, g$ are in certain function spaces. Extending the Lipschitz estimate (1.11) to solutions with Neumann boundary conditions has been a longstanding open problem. The main reason why it is more difficult to deal with solutions with Neumann boundary conditions in Theorem 1.2 than solutions with Dirichlet boundary conditions in [3, 7 ] is that now the boundary conditions in (1.10) are $\varepsilon$-dependent, which causes new difficulties in the estimation of the appropriate boundary correctors. We have overcome this difficulty, in the presence of symmetry, thanks to the Rellich estimates obtained in 22,23. Neumann boundary conditions are important in applications of homogenization (see e.g. [8, 18, 27, 28]). The uniform estimates we establish in this paper can be used to study convergence problems for solutions $u_{\varepsilon}$, eigenfunctions and eigenvalues with Neumann boundary conditions. As an example, let $w_{\varepsilon}(x)=$ $u_{\varepsilon}(x)-u_{0}(x)-\varepsilon \chi\left(\frac{x}{\varepsilon}\right) \nabla u_{0}(x)$, where $\chi$ denotes the matrix of correctors for $\mathcal{L}_{\varepsilon}$ in $\mathbb{R}^{d}$. It can be shown that $w_{\varepsilon}=w_{\varepsilon}^{(1)}+w_{\varepsilon}^{(2)}$, where $\left\|\nabla w_{\varepsilon}^{(1)}\right\|_{L^{p}(\Omega)} \leq C_{p} \varepsilon\left\|\nabla^{2} u_{0}\right\|_{L^{p}(\Omega)}$ for any $1<p<\infty$, and $\left|\nabla w_{\varepsilon}^{(2)}(x)\right| \operatorname{dist}(x, \partial \Omega) \leq C \varepsilon\left\|\nabla u_{0}\right\|_{L^{\infty}(\partial \Omega)}$ for any $x \in \Omega$. We will return to this in a forthcoming publication [20]. 
Let $N_{\varepsilon}(x, y)$ denote the matrix of Neumann functions for $\mathcal{L}_{\varepsilon}$ in $\Omega$ (see Section 5 ). As a consequence of our uniform Hölder and Lipschitz estimates, we obtain the bounds

$$
\begin{aligned}
\left|N_{\varepsilon}(x, y)\right| & \leq \frac{C}{|x-y|^{d-2}}, \\
\left|\nabla_{x} N_{\varepsilon}(x, y)\right|+\left|\nabla_{y} N_{\varepsilon}(x, y)\right| & \leq \frac{C}{|x-y|^{d-1}}, \\
\left|\nabla_{x} \nabla_{y} N_{\varepsilon}(x, y)\right| & \leq \frac{C}{|x-y|^{d}},
\end{aligned}
$$

for $d \geq 3$ (see Section 8). In view of the work of Avellaneda and Lin on homogenization of Poisson's kernel [6], we remark that the techniques we develop in this paper may also be used to establish asymptotics of $N_{\varepsilon}(x, y)$. This line of research, together with the convergence results mentioned above, will be developed in a forthcoming paper [20].

We should mention that the case $p=2$ in Theorem 1.3 is contained in 23 . In fact, for the elliptic system $\mathcal{L}_{\varepsilon}\left(u_{\varepsilon}\right)=0$ in a bounded Lipschitz domain $\Omega$, the Neumann problem with the uniform estimate $\left\|\left(\nabla u_{\varepsilon}\right)^{*}\right\|_{L^{p}(\partial \Omega)} \leq C\left\|\frac{\partial u_{\varepsilon}}{\partial \nu_{\varepsilon}}\right\|_{L^{p}(\partial \Omega)}$ and the Dirichlet problem with the estimate $\left\|\left(u_{\varepsilon}\right)^{*}\right\|_{L^{p}(\partial \Omega)} \leq C\left\|u_{\varepsilon}\right\|_{L^{p}(\partial \Omega)}$, as well as the so-called regularity problem with the estimate $\left\|\left(\nabla u_{\varepsilon}\right)^{*}\right\|_{L^{p}(\partial \Omega)} \leq C\left\|\nabla_{\tan } u_{\varepsilon}\right\|_{L^{p}(\partial \Omega)}$, were solved recently by Kenig and Shen in [23] for $p$ close to 2 (see [19] for references on boundary value problems in Lipschitz domains for elliptic equations with constant coefficients). The results in [23] are proved under the assumption that $A \in \Lambda(\mu, \lambda, \tau)$ and $A^{*}=A$, by the method of layer potentials. In the case of a single equation $(m=1)$, the $L^{p}$ solvabilities of Neumann, Dirichlet, and regularity problems in Lipschitz domains with uniform nontangential maximal function estimates were established in 22 for the sharp ranges of $p$ 's (the result for the Dirichlet problem in Lipschitz domains was obtained earlier by B. Dahlberg [11, using a different approach; see the appendix to 22 for Dahlberg's proof). The results in [22, 23. rely on uniform Rellich estimates $\left\|\frac{\partial u_{\varepsilon}}{\partial \nu_{\varepsilon}}\right\|_{L^{2}(\partial \Omega)} \approx\left\|\nabla_{\tan } u_{\varepsilon}\right\|_{L^{2}(\partial \Omega)}$ for solutions of $\mathcal{L}_{\varepsilon}\left(u_{\varepsilon}\right)=0$ in a Lipschitz domain $\Omega$. We point out that one of the key steps in the proof of Theorem 1.2 uses the Rellich estimate $\left\|\nabla u_{\varepsilon}\right\|_{L^{2}(\partial \Omega)} \leq C\left\|\frac{\partial u_{\varepsilon}}{\partial \nu_{\varepsilon}}\right\|_{L^{2}(\partial \Omega)}$ in a crucial way.

We now describe the key ideas in the proofs of our main results. To show Theorem 1.1 we first establish the uniform boundary Hölder estimate for local solutions,

$$
\left\|u_{\varepsilon}\right\|_{C^{0, \gamma}(B(Q, \rho) \cap \Omega)} \leq C \rho^{-\gamma}\left(f_{B(Q, 2 \rho) \cap \Omega}\left|u_{\varepsilon}\right|^{2} d x\right)^{1 / 2}
$$

for any $\gamma \in(0,1)$, where $\mathcal{L}_{\varepsilon}\left(u_{\varepsilon}\right)=0$ in $B(Q, 3 \rho) \cap \Omega$ and $\frac{\partial u_{\varepsilon}}{\partial \nu_{\varepsilon}}=0$ on $B(Q, 3 \rho) \cap \partial \Omega$ for some $Q \in \partial \Omega$ and $0<\rho<c$. The proof of (1.16) uses a compactness method, which was developed by Lin and Avellaneda in [3, 5, 6] for homogenization problems, with basic ideas originating from the regularity theory in the calculus of variations and minimal surfaces. As in the case of the Dirichlet boundary condition, boundary correctors are not needed for Hölder estimates with the Neumann boundary 
condition. From (1.16) one may deduce the weak reverse Hölder inequality,

$$
\left(f_{B(Q, \rho) \cap \Omega}\left|\nabla u_{\varepsilon}\right|^{p} d x\right)^{1 / p} \leq C_{p}\left(f_{B(Q, 2 \rho) \cap \Omega}\left|\nabla u_{\varepsilon}\right|^{2} d x\right)^{1 / 2}
$$

for any $p>2$. By [15] this implies that $\left\|\nabla u_{\varepsilon}\right\|_{L^{p}(\Omega)} \leq C\|f\|_{L^{p}(\Omega)}$ for $p>2$, if $\mathcal{L}_{\varepsilon}\left(u_{\varepsilon}\right)=\operatorname{div}(f)$ in $\Omega$ and $\frac{\partial u_{\varepsilon}}{\partial \nu_{\varepsilon}}=-n \cdot f$ on $\partial \Omega$. The rest of Theorem 1.1 follows by some duality arguments.

The proof of Theorem 1.2 is much more difficult than that of Theorem 1.1 . Assume that $0 \in \partial \Omega$. After a simple rescaling, the heart of the matter here is to establish the uniform boundary Lipschitz estimate for local solutions,

$$
\left\|\nabla u_{\varepsilon}\right\|_{L^{\infty}(B(0,1) \cap \Omega)} \leq C\left\{\left\|u_{\varepsilon}\right\|_{L^{\infty}(B(0,2) \cap \Omega)}+\|g\|_{C^{\eta}(B(0,2) \cap \partial \Omega)}\right\},
$$

for some $\eta>0$, where $\mathcal{L}_{\varepsilon}\left(u_{\varepsilon}\right)=0$ in $B(0,3) \cap \Omega$ and $\frac{\partial u_{\varepsilon}}{\partial \nu_{\varepsilon}}=g$ on $B(0,3) \cap \partial \Omega$. This problem has been open for more than twenty years, ever since the same estimate was established in 3] for local solutions with the Dirichlet boundary condition $u_{\varepsilon}=0$ in $B(0,3) \cap \partial \Omega$. Our proof of (1.18) also uses the compactness method mentioned above. However, as in the case of the Dirichlet boundary condition, one needs to introduce suitable boundary correctors in order to fully take advantage of the fact that solutions of the homogenized system are in $C^{1, \eta}(B(0,2) \cap \Omega)$. A major technical breakthrough of this paper is the introduction and estimates of such correctors $\Phi_{\varepsilon}=\left(\Phi_{\varepsilon, j}^{\alpha \beta}\right)$, where for each $1 \leq j \leq d$ and $1 \leq \beta \leq m, \Phi_{\varepsilon, j}^{\beta}=\left(\Phi_{\varepsilon, j}^{1 \beta}, \ldots, \Phi_{\varepsilon, j}^{m \beta}\right)$ is the solution to the Neumann problem

$$
\left\{\begin{array}{rlrl}
\mathcal{L}_{\varepsilon}\left(\Phi_{\varepsilon, j}^{\beta}\right) & =0 & & \text { in } \Omega, \\
\frac{\partial}{\partial \nu_{\varepsilon}}\left(\Phi_{\varepsilon, j}^{\beta}\right) & =\frac{\partial}{\partial \nu_{0}}\left(P_{j}^{\beta}\right) & & \text { on } \partial \Omega, \\
\Phi_{\varepsilon, j}^{\beta}(0) & =0 . &
\end{array}\right.
$$

Here $P_{j}^{\beta}=x_{j}(0, \ldots, 1, \ldots, 0)$ with 1 in the $\beta^{\text {th }}$ position and $\frac{\partial w}{\partial \nu_{0}}$ denotes the conormal derivative of $w$ associated with the homogenized operator $\mathcal{L}_{0}$. Note that by the boundary Hölder estimate, $\Phi_{\varepsilon, j}^{\alpha \beta}(x) \rightarrow x_{j} \delta_{\alpha \beta}$ uniformly in $\Omega$ as $\varepsilon \rightarrow 0$. To carry out an elaborate compactness scheme in a similar fashion to that in [3], one needs to prove the uniform Lipschitz estimate for the solution of (1.19),

$$
\left\|\nabla \Phi_{\varepsilon}\right\|_{L^{\infty}(\Omega)} \leq C .
$$

The proof of (1.20) relies on two crucial observations. First, one can use Rellich estimates as well as boundary Hölder estimates to show that

$$
\int_{\partial \Omega}\left|\nabla_{y}\left\{N_{\varepsilon}(x, y)-N_{\varepsilon}(z, y)\right\}\right| d \sigma(y) \leq C,
$$

where $|x-z| \leq c \operatorname{dist}(x, \partial \Omega)$. Secondly, if $w_{\varepsilon}(x)=\Phi_{\varepsilon}(x)-x I-\varepsilon \chi(x / \varepsilon)$, then $\frac{\partial w_{\varepsilon}}{\partial \nu_{\varepsilon}}$ can be represented as a sum of tangential derivatives of $g_{i j}$ with $\left\|g_{i j}\right\|_{L^{\infty}(\partial \Omega)} \leq C \varepsilon$. Since $\mathcal{L}_{\varepsilon}\left(w_{\varepsilon}\right)=0$ in $\Omega$, it follows from these observations as well as interior estimates that $\left|\nabla w_{\varepsilon}(x)\right| \leq C \varepsilon[\operatorname{dist}(x, \partial \Omega)]^{-1}$. This gives the estimate $\left|\nabla \Phi_{\varepsilon}(x)\right| \leq C$, if $\operatorname{dist}(x, \partial \Omega)>\varepsilon$. The remaining case $\operatorname{dist}(x, \partial \Omega) \leq \varepsilon$ follows by a blow-up argument. See Section 7 for details. We note that the symmetry condition $A^{*}=A$ is only neceded for using the Rellich estimates.

With the Lipschitz estimate in Theorem 1.2 at our disposal, Theorem 1.3 for $p>2$ follows from the case $p=2$ (established in [23] for Lipschitz domains), by a 
real-variable method originating in [9] and further developed in [29]-31. The case $1<p<2$ is handled by establishing the $L^{1}$ estimate for solutions with boundary data in the Hardy space $H^{1}(\partial \Omega)$ and then interpolating it with $L^{2}$ estimates, as in the case of the Laplacian [12 (see Section 9). In view of the Lipschitz estimates in 3 for local solutions with Dirichlet boundary condition and the $L^{2}$ estimates in [23, a similar approach also solves the $L^{p}$ regularity problem with the estimate $\left\|\left(\nabla u_{\varepsilon}\right)^{*}\right\|_{L^{p}(\partial \Omega)} \leq C\left\|\nabla_{\tan } u_{\varepsilon}\right\|_{L^{p}(\partial \Omega)}$ in a $C^{1, \alpha}$ domain $\Omega$ for all $1<p<\infty$ (see Section 10). We further note that the same approach works equally well for the exterior domain $\Omega_{-}=\mathbb{R}^{d} \backslash \bar{\Omega}$ and gives the solvabilities of the $L^{p}$ Neumann and regularity problems in $\Omega_{-}$. Consequently, as in the case of the Laplacian on a Lipschitz domain [12, 33, one may use the $L^{p}$ estimates in $\Omega$ and $\Omega_{-}$and the method of layer potentials to show that solutions to the $L^{p}$ Neumann and regularity problems in $C^{1, \alpha}$ domains may be represented by single layer potentials with density functions that are uniformly bounded in $L^{p}$. Similarly, the solutions to the $L^{p}$ Dirichlet problem may be represented by double layer potentials with uniformly $L^{p}$ bounded density functions (see Section 11).

The summation convention will be used throughout this paper. Finally we remark that we shall make little effort to distinguish vector-valued functions or function spaces from their real-valued counterparts. This should be clear from the context.

\section{Homogenization AND WEAK CONVERGENCE}

Let $\mathcal{L}_{\varepsilon}=-\operatorname{div}(A(x / \varepsilon) \nabla)$ with matrix $A(y)$ satisfying (1.2)-(1.3). For each $1 \leq j \leq d$ and $1 \leq \beta \leq m$, let $\chi_{j}^{\beta}=\left(\chi_{j}^{1 \beta}, \ldots, \chi_{j}^{m \beta}\right)$ be the solution of the following cell problem:

$$
\left\{\begin{array}{l}
\mathcal{L}_{1}\left(\chi_{j}^{\beta}\right)=-\mathcal{L}_{1}\left(P_{j}^{\beta}\right) \text { in } \mathbb{R}^{d}, \\
\chi_{j}^{\beta}(y) \text { is periodic with respect to } \mathbb{Z}^{d}, \\
\int_{[0,1]^{d}} \chi_{j}^{\beta} d y=0,
\end{array}\right.
$$

where $P_{j}^{\beta}=P_{j}^{\beta}(y)=y_{j}(0, \ldots, 1, \ldots, 0)$ with 1 in the $\beta^{\text {th }}$ position. The matrix $\chi=\chi(y)=\left(\chi_{j}^{\alpha \beta}(y)\right)$ with $1 \leq j \leq d$ and $1 \leq \alpha, \beta \leq m$ is called the matrix of correctors for $\left\{\mathcal{L}_{\varepsilon}\right\}$.

With the summation convention the first equation in (2.1) may be written as

$$
\frac{\partial}{\partial y_{i}}\left[a_{i j}^{\alpha \beta}+a_{i \ell}^{\alpha \gamma} \frac{\partial}{\partial y_{\ell}}\left(\chi_{j}^{\gamma \beta}\right)\right]=0 \quad \text { in } \mathbb{R}^{d} .
$$

Let $\hat{A}=\left(\hat{a}_{i j}^{\alpha \beta}\right)$, where $1 \leq i, j \leq d, 1 \leq \alpha, \beta \leq m$, and

$$
\hat{a}_{i j}^{\alpha \beta}=\int_{[0,1]^{d}}\left[a_{i j}^{\alpha \beta}+a_{i \ell}^{\alpha \gamma} \frac{\partial}{\partial y_{\ell}}\left(\chi_{j}^{\gamma \beta}\right)\right] d y .
$$

Then $\mathcal{L}_{0}=-\operatorname{div}(\hat{A} \nabla)$ is the so-called homogenized operator associated with $\left\{\mathcal{L}_{\varepsilon}\right\}$ (see [8]). We need the following homogenization result.

Lemma 2.1. Let $\Omega$ be a bounded Lipschitz domain in $\mathbb{R}^{d}$ and let

$$
\operatorname{div}\left[A_{k}\left(x / \varepsilon_{k}\right) \nabla u_{k}\right]=f \in W_{0}^{-1,2}(\Omega) \quad \text { in } \Omega,
$$


where $\varepsilon_{k} \rightarrow 0$ and the matrix $A_{k}(y)$ satisfies (1.2)-(1.3). Suppose that $u_{k} \rightarrow u_{0}$ strongly in $L^{2}(\Omega), \nabla u_{k} \rightarrow \nabla u_{0}$ weakly in $L^{2}(\Omega)$, and $A_{k}\left(x / \varepsilon_{k}\right) \nabla u_{k}$ converges weakly in $L^{2}(\Omega)$. Also assume that the constant matrix $\hat{A_{k}}$, defined by (2.3) (with $A$ replaced by $\left.A_{k}\right)$, converges to $A^{0}$. Then

$$
A_{k}\left(x / \varepsilon_{k}\right) \nabla u_{k} \rightarrow A^{0} \nabla u_{0} \quad \text { weakly in } L^{2}(\Omega)
$$

and $\operatorname{div}\left(A^{0} \nabla u_{0}\right)=f$ in $\Omega$.

Proof. If $A_{k}$ is independent of $k$, this is a classical result in the theory of homogenization (see e.g. [8] or [10]). The general case may be proved by the same energy method. We give a proof here for the sake of completeness.

Let $A_{k}=\left(a_{i j, k}^{\alpha \beta}\right), \hat{A_{k}}=\left(\hat{a}_{i j, k}^{\alpha \beta}\right)$, and $A^{0}=\left(b_{i j}^{\alpha \beta}\right)$. Suppose that

$$
a_{i \ell, k}^{\alpha \gamma}\left(x / \varepsilon_{k}\right) \frac{\partial u_{k}^{\gamma}}{\partial x_{\ell}} \rightarrow p_{i}^{\alpha}(x) \quad \text { weakly in } L^{2}(\Omega) .
$$

Clearly, $\operatorname{div}(P)=f$ in $\Omega$, where $P=\left(p_{i}^{\alpha}\right)$. For $1 \leq j, \ell \leq d, 1 \leq \beta \leq m$, and $k=1,2, \ldots$, write

$$
\begin{array}{r}
a_{i \ell, k}^{\alpha \gamma}\left(x / \varepsilon_{k}\right) \frac{\partial u_{k}^{\gamma}}{\partial x_{\ell}} \cdot \frac{\partial}{\partial x_{i}}\left\{\varepsilon_{k} \chi_{j, k}^{* \alpha \beta}\left(x / \varepsilon_{k}\right)+x_{j} \delta_{\alpha \beta}\right\} \\
=\frac{\partial u_{k}^{\gamma}}{\partial x_{\ell}} \cdot a_{i \ell, k}^{\alpha \gamma} \frac{\partial}{\partial x_{i}}\left\{\varepsilon_{k} \chi_{j, k}^{* \alpha \beta}\left(x / \varepsilon_{k}\right)+x_{j} \delta_{\alpha \beta}\right\},
\end{array}
$$

where $\chi_{k}^{*}=\left(\chi_{j, k}^{* \alpha \beta}\right)$ denotes the matrix of correctors for $\left(\mathcal{L}_{\varepsilon}^{k}\right)^{*}$, the adjoint operator of $\mathcal{L}_{\varepsilon}^{k}=-\operatorname{div}\left(A_{k}(x / \varepsilon) \nabla\right)$. By taking the weak limits on both sides of (2.5) and using a compensated compactness argument (see e.g. Lemma 5.1 in [10]), we obtain

$$
\begin{aligned}
p_{i}^{\alpha}(x) & \cdot \int_{[0,1]^{d}}\left\{\frac{\partial}{\partial y_{i}}\left[\chi_{j, k}^{* \alpha \beta}(y)\right]+\delta_{i j} \delta_{\alpha \beta}\right\} d y \\
= & \frac{\partial u_{0}^{\gamma}}{\partial x_{\ell}} \cdot \lim _{k \rightarrow \infty} \int_{[0,1]^{d}} a_{i \ell, k}^{\alpha \gamma}\left\{\frac{\partial}{\partial y_{i}}\left[\chi_{j, k}^{* \alpha \beta}(y)\right]+\delta_{i j} \delta_{\alpha \beta}\right\} d y .
\end{aligned}
$$

Since

$$
\int_{[0,1]^{d}} a_{i \ell, k}^{\alpha \gamma}(y) \frac{\partial}{\partial y_{i}}\left\{\chi_{j, k}^{* \alpha \beta}(y)\right\} d y=\int_{[0,1]^{d}} a_{j i, k}^{\beta \alpha}(y) \frac{\partial}{\partial y_{i}}\left\{\chi_{\ell, k}^{\alpha \gamma}(y)\right\} d y
$$

(see e.g. [8, p. 122]), it follows that

$$
\begin{aligned}
p_{j}^{\beta}(x) & =\frac{\partial u_{0}^{\gamma}}{\partial x_{\ell}} \cdot \lim _{k \rightarrow \infty} \int_{[0,1]^{d}}\left\{a_{j \ell, k}^{\beta \gamma}(y)+a_{j i, k}^{\beta \alpha} \frac{\partial}{\partial y_{i}}\left[\chi_{\ell, k}^{\alpha \gamma}(y)\right]\right\} d y \\
& =\frac{\partial u_{0}^{\gamma}}{\partial x_{\ell}} \cdot \lim _{k \rightarrow \infty} \hat{a}_{j \ell, k}^{\beta \gamma} \\
& =b_{j \ell}^{\beta \gamma} \cdot \frac{\partial u_{0}^{\gamma}}{\partial x_{\ell}} .
\end{aligned}
$$

In view of (2.4) this finishes the proof.

Let $\psi: \mathbb{R}^{d-1} \rightarrow \mathbb{R}$ be a $C^{1, \alpha_{0}}$ function such that

$$
\psi(0)=|\nabla \psi(0)|=0 \quad \text { and } \quad\|\nabla \psi\|_{C^{\alpha_{0}\left(\mathbb{R}^{d-1}\right)}} \leq M_{0},
$$


where $\alpha_{0} \in(0,1)$ and $M_{0}>0$ will be fixed throughout the paper. For $r>0$, let (2.7)

$$
\begin{aligned}
& D(r)=D(r, \psi)=\left\{\left(x^{\prime}, x_{d}\right) \in \mathbb{R}^{d}:\left|x^{\prime}\right|<r \text { and } \psi\left(x^{\prime}\right)<x_{d}<\psi\left(x^{\prime}\right)+r\right\}, \\
& \widetilde{D}(r)=\widetilde{D}(r, \psi)=\left\{\left(x^{\prime}, x_{d}\right) \in \mathbb{R}^{d}:\left|x^{\prime}\right|<r \text { and } \psi\left(x^{\prime}\right)-r<x_{d}<\psi\left(x^{\prime}\right)+r\right\}, \\
& \Delta(r)=\Delta(r, \psi)=\left\{\left(x^{\prime}, \psi\left(x^{\prime}\right)\right) \in \mathbb{R}^{d}:\left|x^{\prime}\right|<r\right\} .
\end{aligned}
$$

Lemma 2.2. Let $\left\{\psi_{k}\right\}$ be a sequence of $C^{1, \alpha_{0}}$ functions satisfying (2.6). Suppose that $\psi_{k} \rightarrow \psi_{0}$ in $C^{1}\left(\left|x^{\prime}\right|<r\right)$ and that $\left\{\left\|v_{k}\right\|_{L^{2}\left(D\left(r, \psi_{k}\right)\right)}\right\}$ is bounded. Then there exist a subsequence, which we still denote by $\left\{v_{k}\right\}$, and $v_{0} \in L^{2}\left(D\left(r, \psi_{0}\right)\right)$ such that $v_{k} \rightarrow v_{0}$ weakly in $L^{2}(\Omega)$ for any $\Omega \Subset D\left(r, \psi_{0}\right)$.

Proof. Let $w_{k}\left(x^{\prime}, x_{d}\right)=v_{k}\left(x^{\prime}, x_{d}+\psi_{k}\left(x^{\prime}\right)\right)$, defined on

$$
D(r, 0)=\left\{\left(x^{\prime}, x_{d}\right):\left|x^{\prime}\right|<r \text { and } 0<x_{d}<r\right\} .
$$

Since $\left\{w_{k}\right\}$ is bounded in $L^{2}(D(r, 0))$, there exists a subsequence, which we still denote by $\left\{w_{k}\right\}$, such that $w_{k} \rightarrow w_{0}$ weakly in $L^{2}(D(r, 0))$. Let $v_{0}\left(x^{\prime}, x_{d}\right)=$ $w_{0}\left(x^{\prime}, x_{d}-\psi_{0}\left(x^{\prime}\right)\right)$. It is not hard to verify that $v_{k} \rightarrow v_{0}$ weakly in $L^{2}(\Omega)$ if $\Omega \Subset D\left(r, \psi_{0}\right)$.

The following theorem plays an important role in our compactness argument for the Neumann problem. Note that (2.8) is the weak formulation of $\operatorname{div}\left(A_{k}\left(x / \varepsilon_{k}\right) \nabla u_{k}\right)$ $=0$ in $D\left(r, \psi_{k}\right)$ and $\frac{\partial u_{k}}{\partial \nu_{\varepsilon}^{k}}=g_{k}$ on $\Delta\left(r, \psi_{k}\right)$.

Theorem 2.3. Let $\left\{A_{k}(y)\right\}$ be a sequence of matrices satisfying (1.2)-(1.3) and let $\left\{\psi_{k}\right\}$ be a sequence of $C^{1, \alpha_{0}}$ functions satisfying (2.6). Suppose that

$$
\int_{D\left(r, \psi_{k}\right)} A_{k}\left(x / \varepsilon_{k}\right) \nabla u_{k} \cdot \nabla \varphi d x=\int_{\Delta\left(r, \psi_{k}\right)} g_{k} \cdot \varphi d \sigma
$$

for any $\varphi \in C_{0}^{1}\left(\widetilde{D}\left(r, \psi_{k}\right)\right)$, where $\varepsilon_{k} \rightarrow 0$ and

$$
\left\|u_{k}\right\|_{W^{1,2}\left(D\left(r, \psi_{k}\right)\right)}+\left\|g_{k}\right\|_{L^{2}\left(\Delta\left(r, \psi_{k}\right)\right)} \leq C .
$$

Then there exist subsequences of $\left\{\psi_{k}\right\},\left\{u_{k}\right\}$, and $\left\{g_{k}\right\}$, which we still denote by the same notation, and a function $\psi_{0}$ satisfying (2.4), $g_{0} \in L^{2}\left(\Delta\left(r, \psi_{0}\right)\right), u_{0} \in$ $W^{1,2}\left(D\left(r, \psi_{0}\right)\right)$, a constant matrix $A^{0}$ such that

$$
\left\{\begin{array}{l}
\psi_{k} \rightarrow \psi_{0} \text { in } C^{1}\left(\left|x^{\prime}\right|<r\right), \\
g_{k}\left(x^{\prime}, \psi_{k}\left(x^{\prime}\right)\right) \rightarrow g_{0}\left(x^{\prime}, \psi_{0}\left(x^{\prime}\right)\right) \quad \text { weakly in } L^{2}\left(\left|x^{\prime}\right|<r\right), \\
u_{k}\left(x^{\prime}, x_{d}-\psi_{k}\left(x^{\prime}\right)\right) \rightarrow u_{0}\left(x^{\prime}, x_{d}-\psi_{0}\left(x^{\prime}\right)\right) \quad \text { strongly in } L^{2}(D(r, 0)),
\end{array}\right.
$$

and

$$
\int_{D\left(r, \psi_{0}\right)} A^{0} \nabla u_{0} \cdot \nabla \varphi d x=\int_{\Delta\left(r, \psi_{0}\right)} g_{0} \cdot \varphi d \sigma
$$

for any $\varphi \in C_{0}^{1}\left(\widetilde{D}\left(r, \psi_{0}\right)\right)$. Moreover, the matrix $A^{0}$, as the limit of a subsequence of $\left\{\hat{A}_{k}\right\}$, satisfies condition (1.2).

Proof. We first note that (2.10) follows directly from (2.9) by passing to subsequences. To prove (2.11), we fix $\varphi \in C_{0}^{1}\left(\widetilde{D}\left(r, \psi_{0}\right)\right)$. Clearly, if $k$ is sufficiently large, $\varphi \in C_{0}^{1}\left(\widetilde{D}\left(r, \psi_{k}\right)\right)$. It is also easy to check that

$$
\int_{\Delta\left(r, \psi_{k}\right)} g_{k} \cdot \varphi d \sigma \rightarrow \int_{\Delta\left(r, \psi_{0}\right)} g_{0} \cdot \varphi d \sigma .
$$


By passing to a subsequence we may assume that $\hat{A}_{k} \rightarrow A^{0}$. Thus it suffices to show that

$$
\int_{D\left(r, \psi_{k}\right)} A_{k}\left(x / \varepsilon_{k}\right) \nabla u_{k} \cdot \nabla \varphi d x \rightarrow \int_{D\left(r, \psi_{0}\right)} A^{0} \nabla u_{0} \cdot \nabla \varphi d x .
$$

In view of Lemma 2.2 we may assume that $\left\{u_{k}\right\}, \nabla u_{k}$, and $A_{k}\left(x / \varepsilon_{k}\right) \nabla u_{k}$ converge weakly in $L^{2}(\Omega)$ for any $\Omega \Subset D\left(r, \psi_{0}\right)$. As a result, $\left\{u_{k}\right\}$ also converges strongly in $L^{2}(\Omega)$.

Now, given any $\delta>0$, we may choose a Lipschitz domain $\Omega$ such that $\bar{\Omega} \subset$ $D\left(r, \psi_{0}\right)$,

$$
\left|\int_{D\left(r, \psi_{0}\right) \backslash \Omega} A^{0} \nabla u_{0} \cdot \nabla \varphi d x\right|<\delta / 3
$$

and

$$
\left|\int_{D\left(r, \psi_{k}\right) \backslash \Omega} A_{k}\left(x / \varepsilon_{k}\right) \nabla u_{k} \cdot \nabla \varphi d x\right|<\delta / 3
$$

for $k$ sufficiently large. Thus (2.12) would follow if we can show that

$$
\int_{\Omega} A_{k}\left(x / \varepsilon_{k}\right) \nabla u_{k} \cdot \nabla \varphi d x \rightarrow \int_{\Omega} A^{0} \nabla u_{0} \cdot \nabla \varphi d x
$$

This, however, is a direct consequence of Lemma 2.1 since $\operatorname{div}\left(A_{k}\left(x / \varepsilon_{k}\right) \nabla u_{k}\right)=0$ in $\Omega$ by (2.8).

We end this section with the uniform interior gradient estimate, established in 3 by Avellaneda and Lin, for solutions of $\mathcal{L}_{\varepsilon}\left(u_{\varepsilon}\right)=0$. For a ball $B=B(x, r)$ in $\mathbb{R}^{d}$, we let $\rho B=B(x, \rho r)$. We will use $f_{E} f$ to denote $\frac{1}{|E|} \int_{E} f$, the average of $f$ over $E$.

Theorem 2.4. Let $A \in \Lambda(\mu, \lambda, \tau)$. Suppose that $\mathcal{L}_{\varepsilon}\left(u_{\varepsilon}\right)=0$ in $2 B$. Then

$$
\sup _{B}\left|\nabla u_{\varepsilon}\right| \leq C\left(f_{2 B}\left|\nabla u_{\varepsilon}\right|^{2} d x\right)^{1 / 2}
$$

where $C$ depends only on $d, m, \mu, \lambda, \tau$.

\section{Boundary Hölder estimates}

The goal of this section is to establish uniform boundary Hölder estimates for $\mathcal{L}_{\varepsilon}$ under the Neumann boundary condition. Throughout this section we assume that $A \in \Lambda(\mu, \lambda, \tau)$.

Theorem 3.1. Let $\Omega$ be a bounded $C^{1, \alpha_{0}}$ domain. Let $p>0$ and $\gamma \in(0,1)$. Suppose that $\mathcal{L}_{\varepsilon}\left(u_{\varepsilon}\right)=0$ in $B(Q, r) \cap \Omega$ and $\frac{\partial u_{\varepsilon}}{\partial \nu_{\varepsilon}}=g$ on $B(Q, r) \cap \partial \Omega$ for some $Q \in \partial \Omega$ and $0<r<r_{0}$. Then

$$
\sup _{B(Q, r / 2) \cap \Omega}\left|u_{\varepsilon}\right| \leq C\left\{\left(f_{B(Q, r) \cap \Omega}\left|u_{\varepsilon}\right|^{p} d x\right)^{1 / p}+\rho\|g\|_{L^{\infty}(B(Q, r) \cap \partial \Omega)}\right\},
$$

and for $x, y \in B(Q, r / 2) \cap \Omega$,

$$
\left|u_{\varepsilon}(x)-u_{\varepsilon}(y)\right| \leq C\left(\frac{|x-y|}{r}\right)^{\gamma}\left\{\left(f_{B(Q, r) \cap \Omega}\left|u_{\varepsilon}\right|^{p} d x\right)^{1 / p}+\rho\|g\|_{L^{\infty}(B(Q, r) \cap \partial \Omega)}\right\},
$$


where $r_{0}>0$ depends only on $\Omega$ and $C>0$ on $d, m, \mu, \lambda, \tau, p, \gamma$, and $\Omega$.

Let $D(\rho, \psi)$ and $\Delta(\rho, \psi)$ be defined by (2.7). By a change of the coordinate system it will suffice to establish the following.

Theorem 3.2. Let $\gamma \in(0,1)$. Suppose that $\mathcal{L}_{\varepsilon}\left(u_{\varepsilon}\right)=0$ in $D(\rho)$ and $\frac{\partial u_{\varepsilon}}{\partial \nu_{\varepsilon}}=g$ on $\Delta(\rho)$ for some $\rho>0$. Then for any $x, y \in D(\rho / 2)$,

$$
\left|u_{\varepsilon}(x)-u_{\varepsilon}(y)\right| \leq C\left(\frac{|x-y|}{\rho}\right)^{\gamma}\left\{\left(f_{D(\rho)}\left|u_{\varepsilon}\right|^{2}\right)^{1 / 2}+\rho\|g\|_{L^{\infty}(\Delta(\rho))}\right\},
$$

where $D(\rho)=D(\rho, \psi), \Delta(\rho)=D(\rho, \psi)$, and $C>0$ depends only on $d, m, \mu, \lambda, \tau$, $\gamma$, and $\left(\alpha_{0}, M_{0}\right)$ in (2.6).

The proof of Theorem 3.2 uses the compactness method developed in [3,5,6, for homogenization problems. We begin with the well-known Cacciopoli inequality,

$$
\int_{D(s \rho)}\left|\nabla u_{\varepsilon}\right|^{2} d x \leq \frac{C}{(t-s)^{2} \rho^{2}} \int_{D(t \rho)}\left|u_{\varepsilon}\right|^{2} d x+C \rho\|g\|_{L^{2}(\Delta(\rho))}^{2},
$$

where $0<s<t<1, \mathcal{L}_{\varepsilon}\left(u_{\varepsilon}\right)=0$ in $D(\rho)$, and $\frac{\partial u_{\varepsilon}}{\partial \nu_{\varepsilon}}=g$ on $\Delta(\rho)$. The periodicity of $A$ is not needed here.

For a function $u$ defined on $S$, we will use $(\bar{u})_{S}$ (and $f_{S}$ ) to denote its average over $S$.

Lemma 3.3. Fix $\beta \in(0,1)$. There exist $\varepsilon_{0}>0$ and $\theta \in(0,1)$, depending only on $d, m, \mu, \lambda, \tau, \beta$, and $\left(\alpha_{0}, M_{0}\right)$, such that

$$
f_{D(\theta)}\left|u_{\varepsilon}-\left(\overline{u_{\varepsilon}}\right)_{D(\theta)}\right|^{2} \leq \theta^{2 \beta}
$$

whenever $\varepsilon<\varepsilon_{0}, \mathcal{L}_{\varepsilon}\left(u_{\varepsilon}\right)=0$ in $D(1), \frac{\partial u_{\varepsilon}}{\partial \nu_{\varepsilon}}=g$ on $\Delta(1)$,

$$
\|g\|_{L^{\infty}(\Delta(1))} \leq 1, \quad \text { and } \quad f_{D(1)}\left|u_{\varepsilon}-\left(\overline{u_{\varepsilon}}\right)_{D(1)}\right|^{2} \leq 1
$$

Proof. Let $\mathcal{L}_{0}=-\operatorname{div}\left(A^{0} \nabla\right)$, where $A^{0}$ is a constant matrix satisfying (1.2). Let $\beta^{\prime}=(1+\beta) / 2$. By boundary Hölder estimates for solutions of elliptic systems with constant coefficients,

$$
f_{D(r)}\left|w-(\bar{w})_{D(r)}\right|^{2} \leq C_{0} r^{2 \beta^{\prime}} \quad \text { for } 0<r<\frac{1}{4},
$$

whenever $\mathcal{L}_{0}(w)=0$ in $D(1 / 2), \frac{\partial w}{\partial \nu_{0}}=g$ on $\Delta(1 / 2)$,

$$
\|g\|_{L^{\infty}(\Delta(1 / 2))} \leq 1, \quad \text { and } \quad \int_{D(1 / 2)}|w|^{2} \leq|D(1)|,
$$

where $C_{0}$ depends only on $d, m, \beta, \mu$, and $\left(\alpha_{0}, M_{0}\right)$.

Next we choose $\theta \in(0,1 / 4)$ so small that $2 C_{0} \theta^{2 \beta^{\prime}} \leq \theta^{2 \beta}$. We shall show by contradiction that for this $\theta$, there exists $\varepsilon_{0}>0$, depending only on $d, m, \mu, \lambda, \tau$, $\beta$, and $\left(\alpha_{0}, M_{0}\right)$, such that (3.5) holds if $0<\varepsilon<\varepsilon_{0}$ and $u_{\varepsilon}$ satisfies the conditions in Lemma 3.3 . 
To this end let's suppose that there exist sequences $\left\{\varepsilon_{k}\right\},\left\{A_{k}\right\},\left\{u_{\varepsilon_{k}}\right\},\left\{g_{k}\right\}$, and $\left\{\psi_{k}\right\}$ such that $\varepsilon_{k} \rightarrow 0, A_{k} \in \Lambda(\mu, \lambda, \tau), \psi_{k}$ satisfies (2.6),

$$
\begin{gathered}
\left\{\begin{aligned}
\mathcal{L}_{\varepsilon_{k}}^{k}\left(u_{\varepsilon_{k}}\right)=0 & \text { in } D_{k}(1), \\
\frac{\partial u_{\varepsilon_{k}}}{\partial \nu_{\varepsilon_{k}}}=g_{k} & \text { on } \Delta_{k}(1),
\end{aligned}\right. \\
\left\|g_{k}\right\|_{L^{\infty}\left(\Delta_{k}(1)\right) \leq 1, \quad f_{D_{k}(1)}\left|u_{\varepsilon_{k}}-\left(\overline{u_{\varepsilon_{k}}}\right)_{D_{k}(1)}\right|^{2} \leq 1,}
\end{gathered}
$$

and

$$
f_{D_{k}(\theta)}\left|u_{\varepsilon_{k}}-\left(\overline{u_{\varepsilon_{k}}}\right)_{D_{k}(\theta)}\right|^{2}>\theta^{2 \beta},
$$

where $\mathcal{L}_{\varepsilon_{k}}^{k}=-\operatorname{div}\left(A_{k}\left(x / \varepsilon_{k}\right) \nabla\right), D_{k}(r)=D\left(r, \psi_{k}\right)$, and $\Delta_{k}(r)=D\left(r, \psi_{k}\right)$. By subtracting a constant we may assume that $\left(\overline{u_{\varepsilon_{k}}}\right)_{D_{k}(1)}=0$. Thus it follows from (3.9) and the Cacciopoli inequality (3.4) that the norm of $u_{\varepsilon_{k}}$ in $W^{1,2}\left(D_{k}(1 / 2)\right)$ is uniformly bounded. In view of Theorem 2.3 , by passing to subsequences, we may assume that

$$
\left\{\begin{array}{l}
\psi_{k} \rightarrow \psi_{0} \quad \text { in } C^{1}\left(\left|x^{\prime}\right|<1\right) \\
g_{k}\left(x^{\prime}, \psi_{k}\left(x^{\prime}\right)\right) \rightarrow g_{0}\left(x^{\prime}, \psi_{0}\left(x^{\prime}\right)\right) \quad \text { weakly in } L^{2}\left(\left|x^{\prime}\right|<1\right) \\
u_{\varepsilon_{k}}\left(x^{\prime}, x_{d}-\psi_{k}\left(x^{\prime}\right)\right) \rightarrow u_{0}\left(x^{\prime}, x_{d}-\psi_{0}\left(x^{\prime}\right)\right) \quad \text { strongly in } L^{2}(D(1 / 2,0))
\end{array}\right.
$$

and

$$
\begin{cases}\operatorname{div}\left(A^{0} \nabla u_{0}\right)=0 & \text { in } D\left(1 / 2, \psi_{0}\right), \\ \frac{\partial u_{0}}{\partial \nu_{0}}=g_{0} & \text { on } \Delta\left(1 / 2, \psi_{0}\right),\end{cases}
$$

where $A^{0}$ is a constant matrix satisfying (1.2).

Using (3.11) one may verify that

$$
\left|D_{k}(r)\right| \rightarrow\left|D_{0}(r)\right|, \quad\left\|g_{0}\right\|_{L^{\infty}\left(\Delta\left(1, \psi_{0}\right)\right)} \leq 1, \quad\left(\overline{u_{\varepsilon_{k}}}\right)_{D_{k}(r)} \rightarrow\left(\overline{u_{0}}\right)_{D_{0}(r)},
$$

and

$$
\int_{D_{k}(r)}\left|u_{\varepsilon_{k}}-\left(\overline{u_{\varepsilon_{k}}}\right)_{D_{k}(r)}\right|^{2} \rightarrow \int_{D_{0}(r)}\left|u_{0}-\left(\overline{u_{0}}\right)_{D_{0}(r)}\right|^{2}
$$

for any $r \in(0,1 / 2]$, where $D_{0}(r)=D\left(r, \psi_{0}\right)$. It follows that

$$
\begin{aligned}
& \int_{D_{0}(1 / 2)}\left|u_{0}\right|^{2} \leq\left|D_{0}(1)\right|, \\
& \int_{D_{0}(\theta)}\left|u_{0}-\left(\overline{u_{0}}\right)_{D_{0}(\theta)}\right|^{2} \geq \theta^{2 \beta} .
\end{aligned}
$$

In view of (3.6) -3.7 and (3.14) we obtain $\theta^{2 \beta} \leq C_{0} \theta^{2 \beta^{\prime}}$. This contradicts $2 C_{0} \theta^{2 \beta^{\prime}} \leq \theta^{2 \beta}$.

Lemma 3.4. Fix $\beta \in(0,1)$. Let $\varepsilon_{0}, \theta$ be the constants given by Lemma 3.3. Suppose that $\mathcal{L}_{\varepsilon}\left(u_{\varepsilon}\right)=0$ in $D(1, \psi)$ and $\frac{\partial u_{\varepsilon}}{\partial \nu_{\varepsilon}}=g$ on $\Delta(1, \psi)$. Then, if $\varepsilon<\theta^{k-1} \varepsilon_{0}$ for some $k \geq 1$,

$$
f_{D\left(\theta^{k}, \psi\right)}\left|u_{\varepsilon}-\left(\overline{u_{\varepsilon}}\right)_{D\left(\theta^{k}, \psi\right)}\right|^{2} \leq \theta^{2 k \beta} J^{2},
$$


where

$$
J=\max \left\{\left(f_{D(1, \psi)}\left|u_{\varepsilon}-\left(\overline{u_{\varepsilon}}\right)_{D(1, \psi)}\right|^{2}\right)^{1 / 2},\|g\|_{L^{\infty}(\Delta(1, \psi))}\right\} .
$$

Proof. The lemma is proved by induction on $k$. Note that the case $k=1$ is given by Lemma 3.3. Assume now that the lemma holds for some $k \geq 1$. Let $\varepsilon<\theta^{k} \varepsilon_{0}$. We apply Lemma 3.3 to $w(x)=u\left(\theta^{k} x\right)$ in $D\left(1, \psi_{k}\right)$, where $\psi_{k}(x)=\theta^{-k} \psi\left(\theta^{k} x\right)$. Since $\mathcal{L}_{\varepsilon / \theta^{k}}(w)=0$ in $D\left(1, \psi_{k}\right)$, this gives

$$
\begin{aligned}
& f_{D\left(\theta^{k+1}, \psi\right)}\left|u_{\varepsilon}-\left(\overline{u_{\varepsilon}}\right)_{D\left(\theta^{k+1}, \psi\right)}\right|^{2} \\
& \quad=f_{D\left(\theta, \psi_{k}\right)}\left|w-(\bar{w})_{D\left(\theta, \psi_{k}\right)}\right|^{2} \\
& \quad \leq \theta^{2 \beta} \max \left\{f_{D\left(1, \psi_{k}\right)}\left|w-(\bar{w})_{D\left(1, \psi_{k}\right)}\right|^{2}, \theta^{2 k}\|g\|_{\infty}^{2}\right\} \\
& \quad=\theta^{2 \beta} \max \left\{f_{D\left(\theta^{k}, \psi\right)}\left|u_{\varepsilon}-\left(\overline{u_{\varepsilon}}\right)_{D\left(\theta^{k}, \psi\right)}\right|^{2}, \theta^{2 k}\|g\|_{\infty}^{2}\right\} \\
& \leq \theta^{2(k+1) \beta} J^{2},
\end{aligned}
$$

where $\|g\|_{\infty}=\|g\|_{L^{\infty}(\Delta(1, \psi))}$ and the last step follows by the induction assumption. Here we also have used the fact that $\left\|\nabla \psi_{k}\right\|_{C^{\alpha_{0}\left(\mathbb{R}^{d-1}\right)}} \leq\|\nabla \psi\|_{C^{\alpha_{0}\left(\mathbb{R}^{d-1}\right)}} \leq M_{0}$.

Proof of Theorem 3.2. By rescaling we may assume that $\rho=1$. We may also assume that $\varepsilon<\varepsilon_{0}$, since the case $\varepsilon \geq \varepsilon_{0}$ follows directly from classical regularity theory. We may further assume that

$$
\|g\|_{L^{\infty}(\Delta(1))} \leq 1 \quad \text { and } \quad \int_{D(1)}\left|u_{\varepsilon}\right|^{2} \leq 1
$$

Under these assumptions we will show that

$$
f_{D(r)}\left|u_{\varepsilon}-\left(\overline{u_{\varepsilon}}\right)_{D(r)}\right|^{2} \leq C r^{2 \beta}
$$

for any $r \in(0,1 / 4)$. The desired estimate (3.3) with $p=2$ follows from the interior estimate (2.16) and (3.16), using Campanato's characterization of Hölder spaces (see e.g. [16]).

To prove (3.16) we first consider the case $r \geq\left(\varepsilon / \varepsilon_{0}\right)$. Choose $k \geq 0$ so that $\theta^{k+1} \leq r<\theta^{k}$. Then $\varepsilon \leq \varepsilon_{0} r<\varepsilon_{0} \theta^{k}$. It follows from Lemma 3.4 that

$$
\begin{aligned}
& f_{D(r)}\left|u_{\varepsilon}-\left(\overline{u_{\varepsilon}}\right)_{D(r)}\right|^{2} \leq C f_{D\left(\theta^{k}\right)}\left|u_{\varepsilon}-\left(\overline{u_{\varepsilon}}\right)_{D\left(\theta^{k}\right)}\right|^{2} \\
& \leq C \theta^{2 k \beta} \leq C r^{2 \beta} .
\end{aligned}
$$


Next suppose that $r<\left(\varepsilon / \varepsilon_{0}\right)$. Let $w(x)=u_{\varepsilon}(\varepsilon x)$. Then $\mathcal{L}_{1}(w)=0$ in $D\left(\varepsilon_{0}^{-1}, \psi_{\varepsilon}\right)$, where $\psi_{\varepsilon}\left(x^{\prime}\right)=\varepsilon^{-1} \psi\left(\varepsilon x^{\prime}\right)$. By classical regularity we obtain

$$
\begin{aligned}
& f_{D(r, \psi)}\left|u_{\varepsilon}-\left(\overline{u_{\varepsilon}}\right)_{D(r, \psi)}\right|^{2}=f_{D\left(\frac{r}{\varepsilon}, \psi_{\varepsilon}\right)}\left|w-(\bar{w})_{D\left(\frac{r}{\varepsilon}, \psi_{\varepsilon}\right)}\right|^{2} \\
& \leq C\left(\frac{r}{\varepsilon}\right)^{2 \beta} \max \left\{f_{D\left(\frac{1}{\varepsilon_{0}}, \psi_{\varepsilon}\right)}\left|w-(\bar{w})_{D\left(\frac{1}{\varepsilon_{0}}, \psi_{\varepsilon}\right)}\right|^{2}, \varepsilon^{2}\|g\|_{\infty}\right\} \\
& =C\left(\frac{r}{\varepsilon}\right)^{2 \beta} \max \left\{f_{D\left(\frac{\varepsilon}{\varepsilon_{0}}, \psi\right)}\left|u_{\varepsilon}-\left(\overline{u_{\varepsilon}}\right)_{D\left(\frac{\varepsilon}{\varepsilon_{0}}, \psi\right)}\right|^{2}, \varepsilon^{2}\|g\|_{\infty}\right\} \\
& \leq C\left(\frac{r}{\varepsilon}\right)^{2 \beta}\left(\frac{\varepsilon}{\varepsilon_{0}}\right)^{2 \beta}=C \varepsilon_{0}^{-2 \beta} r^{2 \beta},
\end{aligned}
$$

where the last inequality follows from the previous case $r=\left(\varepsilon / \varepsilon_{0}\right)$. This finishes the proof of (3.16) and thus of Theorem 3.2

We are now in a position to give the proof of Theorem 3.1 ,

Proof of Theorem 3.1. By rescaling we may assume that $r=1$. The case $p=2$ follows directly from Theorem 3.2 . To handle the case $0<p<2$, we note that by a simple covering argument, estimate (3.1) for $p=2$ gives

$$
\sup _{B(Q, s) \cap \Omega}\left|u_{\varepsilon}\right| \leq C\left\{\frac{1}{(t-s)^{d}}\left(f_{B(Q, t) \cap \Omega}\left|u_{\varepsilon}\right|^{2}\right)^{1 / 2}+\|g\|_{L^{\infty}(B(Q, 1) \cap \partial \Omega)}\right\},
$$

where $(1 / 4)<s<t<1$. By a convexity argument (see e.g. [14, p. 173]), estimate (3.17) implies that for any $p>0$,

$$
\left(f_{B(Q, 1 / 2) \cap \Omega}\left|u_{\varepsilon}\right|^{2}\right)^{1 / 2} \leq C_{p}\left\{\left(f_{B(Q, 1) \cap \Omega}\left|u_{\varepsilon}\right|^{p}\right)^{1 / p}+\|g\|_{L^{\infty}(B(Q, 1) \cap \partial \Omega)}\right\} .
$$

The case $0<p<2$ now follows from estimate (3.18) and the case $p=2$.

\section{Proof of Theorem 1.1}

Under conditions (1.2) and (1.4), weak solutions to (1.8) exist and are unique, up to an additive constant, provided that the data satisfy the necessary condition $\int_{\Omega} F^{\beta}+\left\langle g^{\beta}, 1\right\rangle=0$ for $1 \leq \beta \leq m$. In this section we will show that the weak solutions satisfy the uniform $W^{1, p}$ estimate in Theorem 1.1.

Our starting point is the following theorem established by J. Geng in [15, using a real-variable method originating in [9] and further developed in 29. 31 .

Theorem 4.1. Let $p>2$ and let $\Omega$ be a bounded Lipschitz domain. Let $\mathcal{L}=$ $-\operatorname{div}(A(x) \nabla)$ be an elliptic operator with coefficients satisfying (1.2). Suppose that

$$
\left\{f_{B \cap \Omega}|\nabla u|^{p}\right\}^{1 / p} \leq C_{0}\left\{f_{2 B \cap \Omega}|\nabla u|^{2}\right\}^{1 / 2}
$$

whenever $u \in W^{1,2}(3 B \cap \Omega), \mathcal{L}(u)=0$ in $3 B \cap \Omega$, and $\frac{\partial u}{\partial \nu}=0$ on $3 B \cap \partial \Omega$. Here $B=B(Q, r)$ is a ball with the property that $0<r<r_{0}$ and either $Q \in \partial \Omega$ 
or $B(Q, 3 r) \subset \Omega$. Then, for any $f \in L^{p}(\Omega)$, the unique (up to constants) $W^{1,2}$ solution to

$$
\left\{\begin{aligned}
\mathcal{L}(u) & =\operatorname{div}(f) & & \text { in } \Omega, \\
\frac{\partial u}{\partial \nu} & =-n \cdot f & & \text { on } \partial \Omega
\end{aligned}\right.
$$

satisfies the estimate

$$
\|\nabla u\|_{L^{p}(\Omega)} \leq C_{p}\|f\|_{L^{p}(\Omega)},
$$

where $C_{p}$ depends only on $d, m, p, \mu, r_{0}, \Omega$, and the constant $C_{0}$ in (4.1).

Now, given $A \in \Lambda(\mu, \lambda, \tau)$ and $p>2$. Let $\Omega$ be a $C^{1, \alpha_{0}}$ domain. Suppose that $\mathcal{L}_{\varepsilon}\left(u_{\varepsilon}\right)=0$ in $3 B \cap \Omega$ and $\frac{\partial u_{\varepsilon}}{\partial_{\nu_{\varepsilon}}}=0$ on $3 B \cap \partial \Omega$. If $3 B \subset \Omega$, the weak reverse Hölder inequality (4.1) for $u_{\varepsilon}$ follows from the interior estimate (2.16). Suppose that $Q \in \partial \Omega$ and $B=B(Q, r)$. We may use the interior estimate and boundary Hölder estimate (3.2) to obtain

$$
\begin{aligned}
\left|\nabla u_{\varepsilon}(x)\right| & \leq C \delta(x)^{-1}\left(f_{B(x, c \delta(x))}\left|u_{\varepsilon}(y)-u_{\varepsilon}(x)\right|^{2} d y\right)^{1 / 2} \\
& \leq C_{\gamma}\left(\frac{r}{\delta(x)}\right)^{\gamma}\left(f_{B(Q, 2 r) \cap \Omega}\left|\nabla u_{\varepsilon}\right|^{2} d y\right)^{1 / 2}
\end{aligned}
$$

for any $\gamma \in(0,1)$ and $x \in B(Q, r) \cap \Omega$, where $\delta(x)=\operatorname{dist}(x, \partial \Omega)$. Choose $\gamma \in(0,1)$ so that $p \gamma<1$. It is easy to see that (4.4) implies

$$
\left(f_{B \cap \Omega}\left|\nabla u_{\varepsilon}\right|^{p}\right)^{1 / p} \leq C_{p}\left(f_{2 B \cap \Omega}\left|\nabla u_{\varepsilon}\right|^{2}\right)^{1 / 2} .
$$

In view of Theorem 4.1 we have proved Theorem 1.1 for the case $p>2, g=0$, and $F=0$.

Lemma 4.2. Suppose $A \in \Lambda(\mu, \lambda, \tau)$. Let $f \in L^{p}(\Omega)$, where $\Omega$ be a bounded $C^{1, \alpha_{0}}$ domain and $1<p<\infty$. Let $u \in W^{1, p}(\Omega)$ be a weak solution to $\mathcal{L}_{\varepsilon}\left(u_{\varepsilon}\right)=\operatorname{div}(f)$ in $\Omega$ and $\frac{\partial u_{\varepsilon}}{\partial \nu_{\varepsilon}}=-n \cdot f$ on $\partial \Omega$. Then $\left\|\nabla u_{\varepsilon}\right\|_{L^{p}(\Omega)} \leq C_{p}\|f\|_{L^{p}(\Omega)}$.

Proof. The case $p>2$ was proved above. Suppose that $1<p<2$. Let $g \in C_{0}^{\infty}(\Omega)$ and let $v_{\varepsilon}$ be a weak solution of $\mathcal{L}_{\varepsilon}^{*}\left(v_{\varepsilon}\right)=\operatorname{div}(g)$ and $\frac{\partial v_{\varepsilon}}{\partial \nu_{\varepsilon}^{*}}=0$ on $\partial \Omega$, where $\mathcal{L}_{\varepsilon}^{*}$ denotes the adjoint of $\mathcal{L}_{\varepsilon}$. Since $A^{*} \in \Lambda(\lambda, \mu, \tau)$ and $p^{\prime}>2$, we have $\left\|\nabla v_{\varepsilon}\right\|_{L^{p^{\prime}}(\Omega)} \leq$ $C\|g\|_{L^{p^{\prime}}(\Omega)}$. Also, note that

$$
\int_{\Omega} f_{i}^{\alpha} \cdot \frac{\partial v_{\varepsilon}^{\alpha}}{\partial x_{i}} d x=\int_{\Omega} a_{i j}^{\alpha \beta}\left(\frac{x}{\varepsilon}\right) \frac{\partial u_{\varepsilon}^{\beta}}{\partial x_{j}} \cdot \frac{\partial v_{\varepsilon}^{\alpha}}{\partial x_{i}} d x=\int_{\Omega} g_{i}^{\alpha} \cdot \frac{\partial u_{\varepsilon}^{\alpha}}{\partial x_{i}} d x
$$

where $f=\left(f_{i}^{\alpha}\right)$ and $g=\left(g_{i}^{\alpha}\right)$. The estimate $\left\|\nabla u_{\varepsilon}\right\|_{L^{p}(\Omega)} \leq C\|f\|_{L^{p}(\Omega)}$ now follows from (4.5) by duality.

Lemma 4.3. Suppose that $A \in \Lambda(\lambda, \mu, \tau)$. Let $g=\left(g^{\alpha}\right) \in B^{-1 / p, p}(\partial \Omega)$, where $\Omega$ is a bounded $C^{1, \alpha_{0}}$ domain, $1<p<\infty$, and $\left\langle g^{\alpha}, 1\right\rangle=0$. Let $u \in W^{1, p}(\Omega)$ be a weak solution to $\mathcal{L}_{\varepsilon}\left(u_{\varepsilon}\right)=0$ in $\Omega$ and $\frac{\partial u_{\varepsilon}}{\partial \nu_{\varepsilon}}=g$ on $\partial \Omega$. Then $\left\|\nabla u_{\varepsilon}\right\|_{L^{p}(\Omega)} \leq$ $C_{p}\|g\|_{B^{-1 / p, p}(\partial \Omega)}$. 
Proof. Let $f \in C_{0}^{\infty}(\Omega)$ and let $v_{\varepsilon}$ be a weak solution to $\mathcal{L}_{\varepsilon}^{*}\left(v_{\varepsilon}\right)=\operatorname{div}(f)$ in $\Omega$ and $\frac{\partial v_{\varepsilon}}{\partial \nu_{\varepsilon}^{*}}=0$ on $\partial \Omega$. Since $A^{*} \in \Lambda(\lambda, \mu, \tau)$, by Lemma 4.2, we have $\left\|\nabla v_{\varepsilon}\right\|_{L^{p^{\prime}}(\Omega)} \leq$ $C\|f\|_{L^{p^{\prime}}(\Omega)}$.

Note that

$$
\int_{\Omega} f_{i}^{\alpha} \cdot \frac{\partial u_{\varepsilon}^{\alpha}}{\partial x_{i}} d x=-\int_{\Omega} a_{i j}^{\alpha \beta}\left(\frac{x}{\varepsilon}\right) \frac{\partial u_{\varepsilon}^{\beta}}{\partial x_{j}} \cdot \frac{\partial v_{\varepsilon}^{\alpha}}{\partial x_{i}} d x=-\left\langle g, v_{\varepsilon}\right\rangle .
$$

Let $E$ be the average of $v_{\varepsilon}$ over $\Omega$. Then

$$
\begin{aligned}
\left|\left\langle g, v_{\varepsilon}\right\rangle\right| & =\left|\left\langle g, v_{\varepsilon}-E\right\rangle\right| \leq\|g\|_{B^{-1 / p, p}(\partial \Omega)}\left\|v_{\varepsilon}-E\right\|_{B^{1 / p, p^{\prime}}(\partial \Omega)} \\
& \leq C\|g\|_{B^{-1 / p, p}(\partial \Omega)}\left\|v_{\varepsilon}-E\right\|_{W^{1, p^{\prime}}(\Omega)} \\
& \leq C\|g\|_{B^{-1 / p, p}(\partial \Omega)}\left\|\nabla v_{\varepsilon}\right\|_{L^{p^{\prime}}(\Omega)} \\
& \leq C\|g\|_{B^{-1 / p, p}(\partial \Omega)}\|f\|_{L^{p^{\prime}}(\Omega)},
\end{aligned}
$$

where we have used a trace theorem for the second inequality and Poincaré inequality for the third. The estimate $\left\|\nabla u_{\varepsilon}\right\|_{L^{p}(\Omega)} \leq C\|g\|_{B^{-1 / p, p}(\partial \Omega)}$ follows from (4.6) (4.7) by duality.

Let $1<q<d$ and $\frac{1}{p}=\frac{1}{q}-\frac{1}{d}$. In the proof of the next lemma, we will need the Sobolev inequality

$$
\left(\int_{\Omega}|u|^{p} d x\right)^{1 / p} \leq C\left(\int_{\Omega}|\nabla u|^{q} d x\right)^{1 / q},
$$

where $u \in W^{1, q}(\Omega)$ and $\int_{\partial \Omega} u=0$.

Lemma 4.4. Suppose that $A \in \Lambda(\mu, \lambda, \tau)$. Let $F \in L^{q}(\Omega)$, where $1<q<d$ and $\Omega$ is a bounded $C^{1, \alpha_{0}}$ domain. Let $u \in W^{1, p}(\Omega)$ be a weak solution to $\mathcal{L}_{\varepsilon}\left(u_{\varepsilon}\right)=F$ in $\Omega$ and $\frac{\partial u_{\varepsilon}}{\partial \nu_{\varepsilon}}=-b$ on $\partial \Omega$, where $\frac{1}{p}=\frac{1}{q}-\frac{1}{d}$ and $b=\frac{1}{|\partial \Omega|} \int_{\Omega} F$. Then $\left\|\nabla u_{\varepsilon}\right\|_{L^{p}(\Omega)} \leq$ $C\|F\|_{L^{q}(\Omega)}$.

Proof. Let $f \in C_{0}^{\infty}(\Omega)$ and let $v_{\varepsilon}$ be a weak solution to $\left(\mathcal{L}_{\varepsilon}\right)^{*}\left(v_{\varepsilon}\right)=\operatorname{div}(f)$ in $\Omega$ and $\frac{\partial v_{\varepsilon}}{\partial \nu_{\varepsilon}}=0$ on $\partial \Omega$. By Lemma 4.2 we have $\left\|\nabla v_{\varepsilon}\right\|_{L^{p^{\prime}}(\Omega)} \leq C\|f\|_{L^{p^{\prime}}(\Omega)}$. Note that

$$
\begin{aligned}
\int_{\Omega} \frac{\partial u_{\varepsilon}^{\alpha}}{\partial x_{i}} \cdot f_{i}^{\alpha} d x & =\int_{\Omega} a_{i j}^{\alpha \beta}\left(\frac{x}{\varepsilon}\right) \frac{\partial u_{\varepsilon}^{\beta}}{\partial x_{j}} \cdot \frac{\partial v_{\varepsilon}^{\alpha}}{\partial x_{i}} d x \\
& =\int_{\Omega} F \cdot v_{\varepsilon} d x-\int_{\partial \Omega} b \cdot v_{\varepsilon} d \sigma \\
& =\int_{\Omega} F\left(v_{\varepsilon}-E\right) d x
\end{aligned}
$$

where $E$ is the average of $v_{\varepsilon}$ over $\partial \Omega$. It follows from (4.9) and Sobolev inequality (4.8) that

$$
\begin{aligned}
\left|\int_{\Omega} \frac{\partial u_{\varepsilon}^{\alpha}}{\partial x_{i}} \cdot f_{i}^{\alpha} d x\right| & \leq\|F\|_{L^{q}(\Omega)}\left\|v_{\varepsilon}-E\right\|_{L^{q^{\prime}}(\Omega)} \\
& \leq C\|F\|_{L^{q}(\Omega)}\left\|\nabla v_{\varepsilon}\right\|_{L^{p^{\prime}}(\Omega)} \\
& \leq C\|F\|_{L^{q}(\Omega)}\|f\|_{L^{p^{\prime}}(\Omega)}
\end{aligned}
$$

By duality this gives $\left\|\nabla u_{\varepsilon}\right\|_{L^{p}(\Omega)} \leq C\|F\|_{L^{q}(\Omega)}$. 
Proof of Theorem 1.1. Let $v_{\varepsilon}$ be a weak solution to $\mathcal{L}_{\varepsilon}\left(v_{\varepsilon}\right)=\operatorname{div}(f)$ in $\Omega$ and $\frac{\partial v_{\varepsilon}}{\partial \nu_{\varepsilon}}=-n \cdot f$ on $\partial \Omega$. Let $w_{\varepsilon}$ be a weak solution to $\mathcal{L}_{\varepsilon}\left(w_{\varepsilon}\right)=F$ in $\Omega$ and $\frac{\partial w_{\varepsilon}}{\partial \nu_{\varepsilon}}=-b$ on $\partial \Omega$, where $b=\frac{1}{|\partial \Omega|} \int_{\Omega} F$. Finally, let $h_{\varepsilon}=u_{\varepsilon}-v_{\varepsilon}-w_{\varepsilon}$. Then $\mathcal{L}_{\varepsilon}\left(h_{\varepsilon}\right)=0$ in $\Omega$ and $\frac{\partial h_{\varepsilon}}{\partial \nu_{\varepsilon}}=g+b$ on $\partial \Omega$. It follows from Lemmas 4.2, 4.3, and 4.4 that

$$
\begin{aligned}
\left\|\nabla u_{\varepsilon}\right\|_{L^{p}(\Omega)} & \leq\left\|\nabla v_{\varepsilon}\right\|_{L^{p}(\Omega)}+\left\|\nabla w_{\varepsilon}\right\|_{L^{p}(\Omega)}+\left\|\nabla h_{\varepsilon}\right\|_{L^{p}(\Omega)} \\
& \leq C\left\{\|f\|_{L^{p}(\Omega)}+\|F\|_{L^{q}(\Omega)}+\|g\|_{B^{-1 / p, p}(\partial \Omega)}\right\},
\end{aligned}
$$

where $q=\frac{p d}{p+d}$ for $p>\frac{d}{d-1}$ and $q>1$ for $1<p \leq \frac{d}{d-1}$. This completes the proof.

\section{A matrix of Neumann functions}

Let $\Gamma_{\varepsilon}(x, y)=\left(\Gamma_{A, \varepsilon}^{\alpha \beta}(x, y)\right)_{m \times m}$ denote the matrix of fundamental solutions of $\mathcal{L}_{\varepsilon}$ in $\mathbb{R}^{d}$, with pole at $y$. Under the assumption $A \in \Lambda(\mu, \lambda, \tau)$, one may use the interior estimate (2.16) to show that for $d \geq 3$,

$$
\left|\Gamma_{\varepsilon}(x, y)\right| \leq C|x-y|^{2-d}
$$

and

$$
\left|\nabla_{x} \Gamma_{\varepsilon}(x, y)\right|+\left|\nabla_{y} \Gamma_{\varepsilon}(x, y)\right| \leq C|x-y|^{1-d},
$$

where $C$ depends only on $d, m, \mu, \lambda$, and $\tau$ (see e.g. [17]; the size estimate (5.1) also follows from [13]). Let $V_{\varepsilon}(x, y)=\left(V_{A, \varepsilon}^{\alpha \beta}(x, y)\right)_{m \times m}$, where for each $y \in \Omega$, $V_{\varepsilon}^{\beta}(x, y)=\left(V_{A, \varepsilon}^{1 \beta}(x, y), \ldots, V_{A, \varepsilon}^{m \beta}(x, y)\right)$ solves

$$
\left\{\begin{aligned}
\mathcal{L}_{\varepsilon}\left(V_{\varepsilon}^{\beta}(\cdot, y)\right) & =0 \quad \text { in } \Omega, \\
\frac{\partial}{\partial \nu_{\varepsilon}}\left\{V_{\varepsilon}^{\beta}(\cdot, y)\right\} & =\frac{\partial}{\partial \nu_{\varepsilon}}\left\{\Gamma_{\varepsilon}^{\beta}(\cdot, y)\right\}+\frac{e^{\beta}}{|\partial \Omega|} \quad \text { on } \partial \Omega, \\
\int_{\partial \Omega} V_{\varepsilon}^{\beta}(x, y) d \sigma(x) & =\int_{\partial \Omega} \Gamma_{\varepsilon}^{\beta}(x, y) d \sigma(x),
\end{aligned}\right.
$$

where $\Gamma_{\varepsilon}^{\beta}(x, y)=\left(\Gamma_{A, \varepsilon}^{1 \beta}(x, y), \ldots, \Gamma_{A, \varepsilon}^{m \beta}(x, y)\right)$ and $e^{\beta}=(0, \ldots, 1, \ldots, 0)$ with 1 in the $\beta^{\text {th }}$ position. We now define

$$
N_{\varepsilon}(x, y)=\left(N_{A, \varepsilon}^{\alpha \beta}(x, y)\right)_{m \times m}=\Gamma_{\varepsilon}(x, y)-V_{\varepsilon}(x, y),
$$

for $x, y \in \Omega$. Note that, if $N_{\varepsilon}^{\beta}(x, y)=\Gamma_{\varepsilon}^{\beta}(x, y)-V_{\varepsilon}^{\beta}(x, y)$,

$$
\left\{\begin{aligned}
\mathcal{L}_{\varepsilon}\left\{N_{\varepsilon}^{\beta}(\cdot, y)\right\} & =e^{\beta} \delta_{y}(x) \quad \text { in } \Omega, \\
\frac{\partial}{\partial \nu_{\varepsilon}}\left\{N_{\varepsilon}^{\beta}(\cdot, y)\right\} & =-e^{\beta}|\partial \Omega|^{-1} \quad \text { on } \partial \Omega \\
\int_{\partial \Omega} N_{\varepsilon}^{\beta}(x, y) d \sigma(x) & =0,
\end{aligned}\right.
$$

where $\delta_{y}(x)$ denotes the Dirac delta function with pole at $y$. We will call $N_{\varepsilon}(x, y)$ the matrix of Neumann functions for $\mathcal{L}_{\varepsilon}$ in $\Omega$.

Lemma 5.1. For any $x, y \in \Omega$, we have

$$
N_{A, \varepsilon}^{\alpha \beta}(x, y)=N_{A^{*}, \varepsilon}^{\beta \alpha}(y, x)
$$

where $A^{*}$ denotes the adjoint of $A$. 
Proof. Note that

$$
\Gamma_{A, \varepsilon}^{\alpha \beta}(x, y)=\Gamma_{A^{*}, \varepsilon}^{\beta \alpha}(y, x), \quad \text { for any } x, y \in \Omega .
$$

Using the Green's representation formula for $\mathcal{L}_{\varepsilon}$ on $\Omega$, (5.3), and (5.5) one may show that

$$
\begin{gathered}
V_{A, \varepsilon}^{\alpha \beta}(x, y)+\Gamma_{A, \varepsilon}^{\alpha \beta}(x, y)-\frac{1}{|\partial \Omega|} \int_{\partial \Omega}\left\{\Gamma_{A, \varepsilon}^{\alpha \beta}(z, y)+\Gamma_{A^{*}, \varepsilon}^{\beta \alpha}(z, x)\right\} d \sigma(z) \\
=\int_{\Omega} a_{i j}^{\gamma \delta}\left(\frac{z}{\varepsilon}\right) \frac{\partial}{\partial z_{i}}\left\{\Gamma_{A^{*}, \varepsilon}^{\gamma \alpha}(z, x)\right\} \cdot \frac{\partial}{\partial z_{j}}\left\{\Gamma_{A, \varepsilon}^{\delta \beta}(z, y)\right\} d z \\
\quad-\int_{\Omega} a_{i j}^{\gamma \delta}\left(\frac{z}{\varepsilon}\right) \frac{\partial}{\partial z_{i}}\left\{V_{A^{*}, \varepsilon}^{\gamma \alpha}(z, x)\right\} \cdot \frac{\partial}{\partial z_{j}}\left\{V_{A, \varepsilon}^{\delta \beta}(z, y)\right\} d z .
\end{gathered}
$$

This gives $V_{A, \varepsilon}^{\alpha \beta}(x, y)=V_{A^{*}, \varepsilon}^{\beta \alpha}(y, x)$ and hence (5.6) .

Theorem 5.2. Let $\Omega$ be a bounded $C^{1, \alpha_{0}}$ domain, and let $A \in \Lambda(\mu, \lambda, \tau)$. Let $x_{0}$, $y_{0}, z_{0} \in \Omega$ be such that $\left|x_{0}-z_{0}\right|<(1 / 4)\left|x_{0}-y_{0}\right|$. Then for any $\gamma \in(0,1)$,

$$
\left\{f_{B\left(y_{0}, \rho / 4\right) \cap \Omega}\left|\nabla_{y}\left\{N_{\varepsilon}\left(x_{0}, y\right)-N_{\varepsilon}\left(z_{0}, y\right)\right\}\right|^{2} d y\right\}^{1 / 2} \leq C \rho^{1-d}\left(\frac{\left|x_{0}-z_{0}\right|}{\rho}\right)^{\gamma}
$$

where $\rho=\left|x_{0}-y_{0}\right|$ and $C$ depends only on $\mu, \lambda, \tau, \gamma$, and $\Omega$.

Proof. Let $f \in C_{0}^{\infty}\left(B\left(y_{0}, \rho / 2\right) \cap \Omega\right)$ and let $\int_{\Omega} f=0$. Let

$$
u_{\varepsilon}(x)=\int_{\Omega} N_{\varepsilon}(x, y) f(y) d y .
$$

Then $\mathcal{L}_{\varepsilon}\left(u_{\varepsilon}\right)=f$ in $\Omega$ and $\frac{\partial u_{\varepsilon}}{\partial \nu_{\varepsilon}}=0$ on $\partial \Omega$. Since $\mathcal{L}_{\varepsilon}\left(u_{\varepsilon}\right)=0$ in $B\left(x_{0}, \rho / 2\right) \cap \Omega$, it follows from the boundary Hölder estimate (3.3) and interior estimates that

$$
\left|u_{\varepsilon}\left(x_{0}\right)-u_{\varepsilon}\left(z_{0}\right)\right| \leq C\left(\frac{\left|x_{0}-z_{0}\right|}{\rho}\right)^{\gamma} \cdot \rho \cdot\left\{f_{B\left(x_{0}, \rho / 2\right) \cap \Omega}\left|\nabla u_{\varepsilon}\right|^{2}\right\}^{1 / 2} .
$$

Let $E$ be the average of $u_{\varepsilon}$ over $B\left(y_{0}, \rho / 2\right) \cap \Omega$. Note that by (1.2),

$$
\begin{aligned}
\mu \int_{\Omega}\left|\nabla u_{\varepsilon}\right|^{2} d x \leq & \left|\int_{\Omega} f \cdot u_{\varepsilon} d x\right|=\left|\int_{B\left(y_{0}, \rho / 2\right) \cap \Omega} f \cdot\left(u_{\varepsilon}-E\right) d x\right| \\
& \leq\|f\|_{L^{2}(\Omega)}\left\|u_{\varepsilon}-E\right\|_{L^{2}\left(B\left(y_{0}, \rho / 2\right) \cap \Omega\right)} \\
& \leq C \rho\|f\|_{L^{2}(\Omega)}\left\|\nabla u_{\varepsilon}\right\|_{L^{2}\left(B\left(y_{0}, \rho / 2\right) \cap \Omega\right)},
\end{aligned}
$$

where we have used the Cauchy and Poincaré inequalities. Hence, $\left\|\nabla u_{\varepsilon}\right\|_{L^{2}(\Omega)} \leq$ $C \rho\|f\|_{L^{2}(\Omega)}$. This, together with (5.9), gives

$$
\left|u_{\varepsilon}\left(x_{0}\right)-u_{\varepsilon}\left(z_{0}\right)\right| \leq C \rho^{2-\frac{d}{2}}\left(\frac{\left|x_{0}-z_{0}\right|}{\rho}\right)^{\gamma}\|f\|_{L^{2}(\Omega)} .
$$

By duality this implies that

$$
\left\{\int_{B\left(y_{0}, \rho / 2\right) \cap \Omega}\left|W(y)-C_{x_{0}, z_{0}}\right|^{2} d y\right\}^{1 / 2} \leq C \rho^{2-\frac{d}{2}}\left(\frac{\left|x_{0}-z_{0}\right|}{\rho}\right)^{\gamma},
$$

where $W(y)=N_{\varepsilon}\left(x_{0}, y\right)-N_{\varepsilon}\left(z_{0}, y\right)$ and $C_{x_{0}, z_{0}}$ is the average of $W$ over $B\left(y_{0}, \rho / 2\right) \cap$ $\Omega$. In view of (5.6) we have $\left(\mathcal{L}_{\varepsilon}\right)^{*}\left(W^{*}\right)=0$ in $B\left(y_{0}, \rho / 2\right) \cap \Omega$ and $\frac{\partial}{\partial \nu_{\varepsilon}^{*}}\left\{W^{*}\right\}=0$ on 
$\partial \Omega$, where $\frac{\partial}{\partial \nu_{\varepsilon}^{*}}$ denotes the conormal derivative associated with $\left(\mathcal{L}_{\varepsilon}\right)^{*}$. The estimate (5.8) now follows from (5.11) by Cacciopoli's inequality (3.4).

Lemma 5.3. Let $V_{\varepsilon}(x, y)$ be defined by (5.3). Suppose $d \geq 3$. Then for any $x, y \in \Omega$,

$$
\left|V_{\varepsilon}(x, y)\right| \leq C[\delta(x)]^{\frac{2-d}{2}}[\delta(y)]^{\frac{2-d}{2}},
$$

where $\delta(x)=\operatorname{dist}(x, \partial \Omega)$

Proof. We begin by fixing $y \in \Omega$ and $1 \leq \beta \leq m$. Let $u_{\varepsilon}(x)=V_{\varepsilon}(x, y)$. In view of (5.3) we have

$$
\left\|\nabla u_{\varepsilon}\right\|_{L^{2}(\Omega)} \leq C\left\|\frac{\partial u_{\varepsilon}}{\partial \nu_{\varepsilon}}\right\|_{W^{-1 / 2,2}(\partial \Omega)} \leq C\left\|\frac{\partial u_{\varepsilon}}{\partial \nu_{\varepsilon}}\right\|_{L^{p}(\partial \Omega)},
$$

where $p=\frac{2(d-1)}{d}$. Note that by (5.2),

$$
\begin{aligned}
\left\|\frac{\partial u_{\varepsilon}}{\partial \nu_{\varepsilon}}\right\|_{L^{p}(\partial \Omega)} & \leq C\left\{\int_{\partial \Omega} \frac{d \sigma(x)}{|x-y|^{p(d-1)}}\right\}^{1 / p}+C|\partial \Omega|^{\frac{1}{p}-1} \\
& \leq C[\delta(y)]^{\frac{2-d}{2}} .
\end{aligned}
$$

Thus we have proved that

$$
\left\|\nabla u_{\varepsilon}\right\|_{L^{2}(\Omega)} \leq C[\delta(y)]^{\frac{2-d}{2}} .
$$

Now, by the interior estimates and the Sobolev inequality (4.8),

$$
\begin{aligned}
\left|u_{\varepsilon}(x)\right| & \leq C\left\{\frac{1}{[\delta(x)]^{d}} \int_{B(x, \delta(x) / 2)}\left|u_{\varepsilon}(z)\right|^{2^{*}} d z\right\}^{1 / 2^{*}} \\
& \leq C[\delta(x)]^{\frac{2-d}{2}}\left\{\left(\int_{\Omega}\left|\nabla u_{\varepsilon}\right|^{2} d x\right)^{1 / 2}+|\Omega|^{\frac{1}{2^{*}}}\left|f_{\partial \Omega} u_{\varepsilon} d \sigma\right|\right\} \\
& \leq C[\delta(x)]^{\frac{2-d}{2}}\left\{[\delta(y)]^{\frac{2-d}{2}}+|\Omega|^{\frac{1}{2^{*}}}\left|f_{\partial \Omega} \Gamma_{\varepsilon}(z, y) d \sigma(z)\right|\right\} \\
& \leq C[\delta(x)]^{\frac{2-d}{2}}[\delta(y)]^{\frac{2-d}{2}}
\end{aligned}
$$

where $2^{*}=\frac{2 d}{d-2}$.

Theorem 5.4. Let $\Omega$ be a bounded $C^{1, \alpha}$ domain in $\mathbb{R}^{d}, d \geq 3$. Suppose that $A \in \Lambda(\mu, \lambda, \tau)$. Then

$$
\left|N_{\varepsilon}(x, y)\right| \leq C|x-y|^{2-d}
$$

and for any $\gamma \in(0,1)$,

$$
\begin{aligned}
& \left|N_{\varepsilon}(x, y)-N_{\varepsilon}(z, y)\right| \leq \frac{C_{\gamma}|x-z|^{\gamma}}{|x-y|^{d-2+\gamma}}, \\
& \left|N_{\varepsilon}(y, x)-N_{\varepsilon}(y, z)\right| \leq \frac{C_{\gamma}|x-z|^{\gamma}}{|x-y|^{d-2+\gamma}},
\end{aligned}
$$

where $|x-z|<(1 / 4)|x-y|$. 
Proof. By Theorem 3.1 we only need to establish the size estimate (5.13). To this end we first note that by Lemma 5.3 .

$$
\left|N_{\varepsilon}(x, y)\right| \leq C\left\{|x-y|^{2-d}+[\delta(x)]^{2-d}+[\delta(y)]^{2-d}\right\} .
$$

Next, let $\rho=|x-y|$. It follows from Theorem 3.1 and (5.15) that

$$
\begin{aligned}
\left|N_{\varepsilon}(x, y)\right| & \leq C\left\{\left\{f_{B(x, \rho / 4) \cap \Omega}\left|N_{\varepsilon}(z, y)\right|^{p} d z\right\}^{1 / p}+\frac{\rho}{|\partial \Omega|}\right\} \\
& \leq C\left\{|x-y|^{2-d}+[\delta(y)]^{2-d}\right\},
\end{aligned}
$$

where we have chosen $p$ so that $p(d-2)<1$. With estimate (5.16) at our disposal, another application of Theorem 3.1 gives

$$
\begin{aligned}
\left|N_{\varepsilon}(x, y)\right| & \leq C\left\{\left\{f_{B(y, \rho / 4) \cap \Omega}\left|N_{\varepsilon}(x, z)\right|^{p} d z\right\}^{1 / p}+\rho^{2-d}\right\} \\
& \leq C|x-y|^{2-d} .
\end{aligned}
$$

This finishes the proof.

Remark 5.5. If $m=1$ and $d \geq 3$, the size estimate (5.13) and Hölder estimate (5.14) for some $\gamma>0$ were established in [21] for divergence form elliptic operators with bounded measurable coefficients in bounded star-like Lipschitz domains.

Remark 5.6. Suppose that $d \geq 3$. The matrix of Neumann functions for the exterior domain $\Omega_{-}=\mathbb{R}^{d} \backslash \bar{\Omega}$ may be constructed in a similar fashion. Indeed, let $N_{\varepsilon}^{-}(x, y)=$ $\Gamma_{\varepsilon}(x, y)-V_{\varepsilon}^{-}(x, y)$, where $V_{\varepsilon}^{-}(x, y)$ is chosen so that for each $y \in \Omega_{-}$,

$$
\left\{\begin{aligned}
\mathcal{L}_{\varepsilon}\left\{N_{\varepsilon}^{-}(\cdot, y)\right\} & =\delta_{y}(x) I \quad \text { in } \Omega, \\
\frac{\partial}{\partial \nu_{\varepsilon}}\left\{N_{\varepsilon}^{-}(\cdot, y)\right\} & =0 \quad \text { on } \partial \Omega, \\
N_{\varepsilon}^{-}(x, y) & =O\left(|x-y|^{2-d}\right) \quad \text { as }|x| \rightarrow \infty
\end{aligned}\right.
$$

where $I$ is the $m \times m$ identity matrix. The estimates in Theorem 5.4 continue to hold for $N_{\varepsilon}^{-}(x, y)$.

Remark 5.7. If $d=2$, the matrix of Neumann functions may be defined as follows. Choose $B(0, R)$ such that $\Omega \subset B(0, R / 2)$. Let $G_{\varepsilon}(x, y)$ be the Green's function for $\mathcal{L}_{\varepsilon}$ in $B(0, R)$. Define $N_{\varepsilon}(x, y)=G_{\varepsilon}(x, y)-V_{\varepsilon}(x, y)$, where $V_{\varepsilon}(x, y)$ is the solution to (5.3), but with $\Gamma_{\varepsilon}(x, y)$ replaced by $G_{\varepsilon}(x, y)$. Theorem 5.2 continues to hold for $d=2$. One may modify the argument in the proof of Lemma 5.3 to show that

$$
\left|V_{\varepsilon}(x, y)\right| \leq C_{\gamma}[\delta(x)]^{-\gamma}[\delta(y)]^{-\gamma}
$$

for any $\gamma>0$. In view of the proof of Theorem 5.4 and the estimate $\left|G_{\varepsilon}(x, y)\right| \leq$ $C\{1+|\ln | x-y||\}$ in $\left[\underline{3}\right.$, this gives $\left|N_{\varepsilon}(x, y)\right| \leq C_{\gamma}|x-y|^{-\gamma}$ for any $\gamma>0$. 


\section{Correctors for Neumann boundary CONDitions}

Let $\Phi_{\varepsilon}=\left(\Phi_{\varepsilon, j}^{\alpha \beta}\right)$, where for each $1 \leq j \leq d$ and $1 \leq \beta \leq m, \Phi_{\varepsilon, j}^{\beta}=\left(\Phi_{\varepsilon, j}^{1 \beta}, \ldots, \Phi_{\varepsilon, j}^{m \beta}\right)$ is a solution to the Neumann problem

$$
\left\{\begin{aligned}
\mathcal{L}_{\varepsilon}\left(\Phi_{\varepsilon, j}^{\beta}\right) & =0 & & \text { in } \Omega, \\
\frac{\partial}{\partial \nu_{\varepsilon}}\left(\Phi_{\varepsilon, j}^{\beta}\right) & =\frac{\partial}{\partial \nu_{0}}\left(P_{j}^{\beta}\right) & & \text { on } \partial \Omega .
\end{aligned}\right.
$$

Here $P_{j}^{\beta}=P_{j}^{\beta}(x)=x_{j}(0, \ldots, 1, \ldots, 0)$ with 1 in the $\beta^{\text {th }}$ position. In the study of boundary estimates for Neumann boundary conditions, the function $\Phi_{\varepsilon}(x)-x$ plays a similar role as $\varepsilon \chi\left(\frac{x}{\varepsilon}\right)$ for interior estimates. The goal of this section is to prove the following uniform Lipschitz estimate of $\Phi_{\varepsilon}$.

Theorem 6.1. Let $\Omega$ be a $C^{1, \alpha_{0}}$ domain. Suppose that $A \in \Lambda(\mu, \lambda, \tau)$ and $A^{*}=A$. Then

$$
\left\|\nabla \Phi_{\varepsilon}\right\|_{L^{\infty}(\Omega)} \leq C
$$

where $C$ depends only on $d, m, \mu, \lambda, \tau$, and $\Omega$.

Our proof of Theorem 6.1 uses the uniform $L^{2}$ Rellich estimate for Neumann problem:

$$
\int_{\partial \Omega}\left|\nabla u_{\varepsilon}\right|^{2} d \sigma \leq C \int_{\partial \Omega}\left|\frac{\partial u_{\varepsilon}}{\partial \nu_{\varepsilon}}\right|^{2} d \sigma
$$

for solutions of $\mathcal{L}_{\varepsilon}\left(u_{\varepsilon}\right)=0$ in $\Omega$. We mention that (6.3) as well as the uniform $L^{2}$ Rellich estimate for the regularity of Dirichlet problem

$$
\int_{\partial \Omega}\left|\nabla u_{\varepsilon}\right|^{2} d \sigma \leq C \int_{\partial \Omega}\left|\nabla_{\tan } u_{\varepsilon}\right|^{2} d \sigma
$$

was established by Kenig and Shen in 23 . under the assumption that $\Omega$ is Lipschitz, $A \in \Lambda(\mu, \lambda, \tau)$, and $A=A^{*}$ (also see [22] for the case of the elliptic equation). The constant $C$ in (6.3) - 6.4) depends only on $d, m, \mu, \lambda, \tau$, and the Lipschitz character of $\Omega$.

Lemma 6.2. Let $\Omega$ and $\mathcal{L}$ satisfy the same assumptions as in Theorem 6.1. Suppose that $\mathcal{L}_{\varepsilon}\left(u_{\varepsilon}\right)=0$ in $\Omega, \frac{\partial u_{\varepsilon}}{\partial \nu_{\varepsilon}}=g$ on $\partial \Omega$, and

$$
g=\sum_{i, j}\left(n_{i} \frac{\partial}{\partial x_{j}}-n_{j} \frac{\partial}{\partial x_{i}}\right) g_{i j},
$$

where $g_{i j} \in C^{1}(\partial \Omega)$ and $n=\left(n_{1}, \ldots, n_{d}\right)$ denotes the unit outward normal to $\partial \Omega$. Then

$$
\left|\nabla u_{\varepsilon}(x)\right| \leq \frac{C}{\delta(x)} \sum_{i, j}\left\|g_{i j}\right\|_{L^{\infty}(\partial \Omega)},
$$

for any $x \in \Omega$, where $\delta(x)=\operatorname{dist}(x, \partial \Omega)$.

Proof. By the interior estimate (2.16) we only need to show that

$$
\left|u_{\varepsilon}(x)-u_{\varepsilon}(z)\right| \leq C \sum_{i, j}\left\|g_{i j}\right\|_{L^{\infty}(\partial \Omega)},
$$


where $|x-z| \leq c r$ and $r=\delta(x)$. Let $N_{\varepsilon}(x, y)$ denote the matrix of Neumann functions for $\mathcal{L}_{\varepsilon}$ on $\Omega$. Note that

$$
\begin{aligned}
u_{\varepsilon}(x)-u_{\varepsilon}(z) & =\int_{\partial \Omega}\left\{N_{\varepsilon}(x, y)-N_{\varepsilon}(z, y)\right\} g(y) d \sigma(y) \\
& =\int_{\partial \Omega}\left\{N_{\varepsilon}(x, y)-N_{\varepsilon}(z, y)\right\} \sum_{i, j}\left(n_{i} \frac{\partial}{\partial y_{j}}-n_{j} \frac{\partial}{\partial y_{i}}\right) g_{i j}(y) d \sigma(y) \\
& =-\sum_{i, j} \int_{\partial \Omega}\left(n_{i} \frac{\partial}{\partial y_{j}}-n_{j} \frac{\partial}{\partial y_{i}}\right)\left\{N_{\varepsilon}(x, y)-N_{\varepsilon}(z, y)\right\} \cdot g_{i j}(y) d \sigma(y),
\end{aligned}
$$

where we have used the fact that $n_{i} \frac{\partial}{\partial y_{j}}-n_{j} \frac{\partial}{\partial y_{i}}$ is a tangential derivative on $\partial \Omega$. Consequently it suffices to show that

$$
\int_{\partial \Omega}\left|\nabla_{y}\left\{N_{\varepsilon}(x, y)-N_{\varepsilon}(z, y)\right\}\right| d \sigma(y) \leq C,
$$

if $|x-z| \leq c r$ and $r=\delta(x)$.

Let $Q \in \partial \Omega$ so that $|x-Q|=\operatorname{dist}(x, \partial \Omega)$. By translation and rotation we may assume that $Q=0$ and

$$
\begin{aligned}
\Omega & \cap\left\{\left(x^{\prime}, x_{d}\right):\left|x^{\prime}\right|<8 c r \text { and }\left|x_{d}\right|<8 c r\right\} \\
& =\left\{\left(x^{\prime}, x_{d}\right):\left|x^{\prime}\right|<8 c r \text { and } \psi\left(x^{\prime}\right)<x_{d}<8 c r\right\}
\end{aligned}
$$

where $\psi(0)=|\nabla \psi(0)|=0$ and $c$ is sufficiently small. To establish (6.7) we will show that

$$
\int_{|y| \leq c r}\left|\nabla_{y}\left\{N_{\varepsilon}(x, y)-N_{\varepsilon}(z, y)\right\}\right| d \sigma(y) \leq C
$$

and that there exists $\beta>0$ such that for $c r<\rho<r_{0}$,

$$
\int_{|y-P| \leq c \rho}\left|\nabla_{y}\left\{N_{\varepsilon}(x, y)-N_{\varepsilon}(z, y)\right\}\right| d \sigma(y) \leq C\left(\frac{r}{\rho}\right)^{\beta},
$$

where $P \in \partial \Omega$ and $|P|=\rho$. The estimate (6.7) follows from (6.8) and (6.9) by a simple covering argument.

To see (6.8) we let

$$
S(t)=\left\{\left(x^{\prime}, x_{d}\right):\left|x^{\prime}\right|<t \text { and } \psi\left(x^{\prime}\right)<x_{d}<\psi\left(x^{\prime}\right)+c t\right\} .
$$

Note that by Cauchy inequality, for $t \in(\mathrm{cr}, 2 \mathrm{cr})$,

$$
\begin{aligned}
& \left\{\int_{|y| \leq c r}\left|\nabla_{y}\left\{N_{\varepsilon}(x, y)-N_{\varepsilon}(z, y)\right\}\right| d \sigma(y)\right\}^{2} \\
& \leq C r^{d-1} \int_{\partial S(t)}\left|\nabla_{y}\left\{N_{\varepsilon}(x, y)-N_{\varepsilon}(z, y)\right\}\right|^{2} d \sigma(y) \\
& \quad \leq C r^{d-1} \int_{\partial S(t)}\left|\frac{\partial}{\partial \nu_{\varepsilon}^{*}}\left\{N_{\varepsilon}(x, y)-N_{\varepsilon}(z, y)\right\}\right|^{2} d \sigma(y),
\end{aligned}
$$

where we have used the Rellich estimate (6.3) for the last inequality. Since

$$
\frac{\partial}{\partial \nu_{\varepsilon}^{*}(y)}\left\{N_{\varepsilon}(x, y)-N_{\varepsilon}(z, y)\right\}=0 \quad \text { in } \partial \Omega,
$$


we may integrate both sides of (6.10) in $t$ over $(c r, 2 c r)$ to obtain

$$
\begin{aligned}
& \left\{\int_{|y| \leq c r}\left|\nabla_{y}\left\{N_{\varepsilon}(x, y)-N_{\varepsilon}(z, y)\right\}\right| d \sigma(y)\right\}^{2} \\
& \quad \leq C r^{d-2} \int_{S(2 c r)}\left|\nabla_{y}\left\{N_{\varepsilon}(x, y)-N_{\varepsilon}(z, y)\right\}\right|^{2} d y .
\end{aligned}
$$

The desired estimate (6.8) now follows from estimate (5.8).

The proof of (6.9) is similar to that of (6.8). Indeed, an analogous argument gives

$$
\begin{aligned}
& \left\{\int_{|y-P| \leq c \rho}\left|\nabla_{y}\left\{N_{\varepsilon}(x, y)-N_{\varepsilon}(z, y)\right\}\right|^{2} d \sigma(y)\right\}^{2} \\
& \leq C \rho^{d-2} \int_{|y-P| \leq 2 c \rho}\left|\nabla_{y}\left\{N_{\varepsilon}(x, y)-N_{\varepsilon}(z, y)\right\}\right|^{2} d y \\
& \leq C\left(\frac{r}{\rho}\right)^{2 \gamma} .
\end{aligned}
$$

This completes the proof.

Let $\Psi_{\varepsilon}=\left(\Psi_{\varepsilon, j}^{\alpha \beta}(x)\right)$, where $1 \leq j \leq d, 1 \leq \alpha, \beta \leq m$, and

$$
\Psi_{\varepsilon, j}^{\alpha \beta}(x)=\Phi_{\varepsilon, j}^{\alpha \beta}(x)-x_{j} \delta_{\alpha \beta}-\varepsilon \chi_{j}^{\alpha \beta}\left(\frac{x}{\varepsilon}\right) .
$$

Lemma 6.3. Suppose that $\Omega$ and $\mathcal{L}$ satisfy the same conditions as in Theorem 6.1, Then

$$
\left|\nabla \Psi_{\varepsilon}(x)\right| \leq \frac{C \varepsilon}{\delta(x)} \quad \text { for any } x \in \Omega
$$

Proof. Fix $1 \leq \ell \leq d$ and $1 \leq \gamma \leq m$. Let $w=\left(w^{1}, \ldots, w^{m}\right)=\left(\Psi_{\varepsilon, \ell}^{1 \gamma}, \ldots, \Psi_{\varepsilon, \ell}^{m \gamma}\right)$. Note that $\mathcal{L}_{\varepsilon}(w)=0$ in $\Omega$. In view of Lemma 6.2 it suffices to show that there exists $g_{i j} \in C^{1}(\partial \Omega)$ such that

$$
\left\{\begin{array}{l}
\frac{\partial w}{\partial \nu_{\varepsilon}}=\sum_{i, j}\left(n_{i} \frac{\partial}{\partial x_{j}}-n_{j} \frac{\partial}{\partial x_{i}}\right) g_{i j} \\
\left\|g_{i j}\right\|_{L^{\infty}(\partial \Omega)} \leq C \varepsilon
\end{array}\right.
$$

To this end we observe that by the definition of $\Phi_{\varepsilon, j}^{\alpha \beta}$ in (6.1),

$$
\begin{aligned}
\left(\frac{\partial w}{\partial \nu_{\varepsilon}}\right)^{\alpha} & =n_{i} a_{i j}^{\alpha \beta}\left(\frac{x}{\varepsilon}\right) \frac{\partial}{\partial x_{j}}\left\{\Phi_{\varepsilon, \ell}^{\beta \gamma}\right\}-n_{i} a_{i j}^{\alpha \beta}\left(\frac{x}{\varepsilon}\right) \frac{\partial}{\partial x_{j}}\left\{x_{\ell} \delta_{\beta \gamma}+\varepsilon \chi_{\ell}^{\beta \gamma}\left(\frac{x}{\varepsilon}\right)\right\} \\
& =n_{i} \hat{a}_{i j}^{\alpha \beta} \frac{\partial}{\partial x_{j}}\left\{x_{\ell} \delta_{\beta \gamma}\right\}-n_{i} a_{i j}^{\alpha \beta}\left(\frac{x}{\varepsilon}\right) \frac{\partial}{\partial x_{j}}\left\{x_{\ell} \delta_{\beta \gamma}+\varepsilon \chi_{\ell}^{\beta \gamma}\left(\frac{x}{\varepsilon}\right)\right\} \\
& =n_{i} \hat{a}_{i \ell}^{\alpha \gamma}-n_{i} a_{i j}^{\alpha \beta}\left(\frac{x}{\varepsilon}\right)\left\{\delta_{j \ell} \delta_{\beta \gamma}+\frac{\partial \chi_{\ell}^{\beta \gamma}}{\partial x_{j}}\left(\frac{x}{\varepsilon}\right)\right\},
\end{aligned}
$$

where $\hat{a}_{i j}^{\alpha \beta}$ are the homogenized coefficients defined by (2.3). Let

$$
H_{i \ell}^{\alpha \gamma}(y)=\hat{a}_{i \ell}^{\alpha \gamma}-a_{i j}^{\alpha \beta}(y)\left\{\delta_{j \ell} \delta_{\beta \gamma}+\frac{\partial \chi_{\ell}^{\beta \gamma}}{\partial y_{j}}(y)\right\} .
$$


It follows from the definition of $\hat{a}_{i \ell}^{\alpha \gamma}$ that

$$
\int_{[0,1]^{d}} H_{i \ell}^{\alpha \gamma}(y) d y=0 .
$$

Thus we may solve the Poisson equation on $[0,1]^{d}$ with periodic boundary conditions,

$$
\left\{\begin{array}{l}
\Delta U_{i \ell}^{\alpha \gamma}=H_{i \ell}^{\alpha \gamma} \text { in } \mathbb{R}^{d}, \\
U_{i \ell}^{\alpha \gamma}(y) \text { is periodic with respect to } \mathbb{Z}^{d} .
\end{array}\right.
$$

Since $A(y)$ and $\nabla \chi(y)$ are Hölder continuous, $\nabla^{2} U_{i \ell}^{\alpha \gamma}$ is Hölder continuous. In particular, we have $\left\|\nabla U_{i \ell}^{\alpha \gamma}\right\|_{\infty} \leq C$, where $C$ depends only on $\mu, \lambda$, and $\tau$.

Now let

Then

$$
F_{i \ell k}^{\alpha \gamma}(y)=\frac{\partial}{\partial y_{k}}\left\{U_{i \ell}^{\alpha \gamma}(y)\right\}
$$

and hence

$$
H_{i \ell}^{\alpha \gamma}(y)=\frac{\partial}{\partial y_{k}}\left\{F_{i \ell k}^{\alpha \gamma}(y)\right\}
$$

$$
\begin{aligned}
\left(\frac{\partial w}{\partial \nu_{\varepsilon}}\right)^{\alpha} & =n_{i}(x) H_{i \ell}^{\alpha \gamma}\left(\frac{x}{\varepsilon}\right) \\
& =n_{i}(x) \frac{\partial}{\partial x_{k}}\left\{\varepsilon F_{i \ell k}^{\alpha \gamma}\left(\frac{x}{\varepsilon}\right)\right\} .
\end{aligned}
$$

We claim that

$$
\frac{\partial}{\partial y_{i}}\left\{F_{i \ell k}^{\alpha \gamma}(y)\right\}=0
$$

Assume the claim is true. We may then write

$$
\left(\frac{\partial w}{\partial \nu_{\varepsilon}}\right)^{\alpha}=n_{i}(x) \frac{\partial}{\partial x_{k}}\left\{\varepsilon F_{i \ell k}^{\alpha \gamma}\left(\frac{x}{\varepsilon}\right)\right\}-n_{k}(x) \frac{\partial}{\partial x_{i}}\left\{\varepsilon F_{i \ell k}^{\alpha \gamma}\left(\frac{x}{\varepsilon}\right)\right\} \quad \text { on } \partial \Omega
$$

Since $\left\|\varepsilon F_{i \ell k}^{\alpha \gamma}(x / \varepsilon)\right\|_{\infty} \leq C \varepsilon$, we obtain the desired (6.14).

Finally, to show (6.18), we observe that

$$
\frac{\partial}{\partial y_{i}}\left\{H_{i \ell}^{\alpha \gamma}(y)\right\}=0 \quad \text { in } \mathbb{R}^{d}
$$

which follows directly from (2.2). In view of (6.16) this implies that $\frac{\partial}{\partial y_{i}}\left\{U_{i \ell}^{\alpha \gamma}(y)\right\}$ is harmonic in $\mathbb{R}^{d}$. Since it is also periodic, we may deduce that $\frac{\partial}{\partial y_{i}}\left\{U_{i \ell}^{\alpha \gamma}(y)\right\}$ is constant. As a result,

$$
\frac{\partial}{\partial y_{i}}\left\{F_{i \ell k}^{\alpha \gamma}(y)\right\}=\frac{\partial^{2}}{\partial y_{k} \partial y_{i}}\left\{U_{i \ell}^{\alpha \gamma}(y)\right\}=0 \quad \text { in } \mathbb{R}^{d}
$$

This completes the proof of Lemma 6.2

Proof of Theorem 6.1. It follows from (6.12) and (6.13) that

$$
\left|\nabla \Phi_{\varepsilon}(x)\right| \leq C+\frac{C \varepsilon}{\delta(x)} \quad \text { for any } x \in \Omega .
$$

This implies that $\left|\nabla \Phi_{\varepsilon}(x)\right| \leq C$ if $\delta(x) \geq c \varepsilon$. To estimate $\left|\nabla \Phi_{\varepsilon}(x)\right|$ for $x$ with $\delta(x) \leq c \varepsilon$, we use a standard blow-up argument. 
Fix $j$ and $\beta$. Let $w(x)=\varepsilon^{-1} \Phi_{\varepsilon, j}^{\beta}(\varepsilon x)$. Then $\mathcal{L}_{1}(w)=0$ and

$$
\frac{\partial w}{\partial \nu_{1}}=\frac{\partial \Phi_{\varepsilon, j}^{\beta}}{\partial \nu_{\varepsilon}}(\varepsilon x)=n_{i}(\varepsilon x) \hat{a}_{i j}^{\alpha \beta} .
$$

Since $\Omega$ is a $C^{1, \alpha_{0}}$ domain, its normal $n(x)$ is Hölder continuous. Thus, by the classical regularity results for the Neumann problem with data in Hölder spaces,

$$
\left\|\nabla \Phi_{\varepsilon}\right\|_{L^{\infty}(B(Q, \varepsilon) \cap \Omega)} \leq C+C\left\{\frac{1}{\varepsilon^{d}} \int_{B(Q, 2 \varepsilon) \cap \Omega}\left|\nabla \Phi_{\varepsilon}\right|^{p} d x\right\}^{1 / p}
$$

for any $p>0$, where $Q \in \partial \Omega$ and $C$ depends only on $d, m, p, \mu, \lambda, \tau$, and $\Omega$. We remark that estimate (6.21) with $p=2$ is well known and the case $0<p<2$ follows from the case $p=2$ by a convexity argument. Finally, it follows from (6.20) and (6.21) with $p<1$ that

$$
\left\|\nabla \Phi_{\varepsilon}\right\|_{L^{\infty}(B(Q, \varepsilon) \cap \Omega)} \leq C .
$$

This finishes the proof of Theorem 6.1

Remark 6.4. Fix $\eta \in C_{0}^{\infty}\left(\mathbb{R}^{d-1}\right)$ so that $\eta\left(x^{\prime}\right)=1$ for $\left|x^{\prime}\right| \leq 2$ and $\eta\left(x^{\prime}\right)=0$ for $\left|x^{\prime}\right| \geq 3$. For any function $\psi$ satisfying the condition (2.6), we may construct a bounded $C^{1, \alpha_{0}}$ domain $\Omega_{\psi}$ in $\mathbb{R}^{d}$ with the properties

$$
\begin{aligned}
& D_{\psi \eta}(4) \subset \Omega_{\psi} \subset\left\{\left(x^{\prime}, x_{d}\right):\left|x^{\prime}\right|<8 \text { and }\left|x_{d}\right|<8\left(M_{0}+1\right)\right\}, \\
& \left\{\left(x^{\prime},(\psi \eta)\left(x^{\prime}\right)\right):\left|x^{\prime}\right|<4\right\} \subset \partial \Omega_{\psi} .
\end{aligned}
$$

Clearly, the domain $\Omega_{\psi}$ can be constructed in such a way that $\Omega_{\psi} \backslash\left\{\left(x^{\prime},(\psi \eta)\left(x^{\prime}\right)\right)\right.$ : $\left.\left|x^{\prime}\right| \leq 4\right\}$ depends only on $M_{0}$.

Let $\Phi_{\varepsilon}(x)=\Phi_{\varepsilon}\left(x, \Omega_{\psi}, A\right)$ be the matrix of functions satisfying (6.1) with $\Omega=\Omega_{\psi}$ and $\Phi_{\varepsilon}(0)=0$. It follows from Theorem 6.1 that $\left\|\nabla \Phi_{\varepsilon}\right\|_{L^{\infty}(\Omega)} \leq C$, where $C$ depends only on $d, m, \mu, \lambda, \tau$, and $\left(\alpha_{0}, M_{0}\right)$.

\section{BOUndaRy Lipschitz ESTIMATES}

In this section we establish the uniform boundary Lipschitz estimate under the assumption that $A \in \Lambda(\mu, \lambda, \tau)$ and $A^{*}=A$.

Theorem 7.1. Let $\Omega$ be a bounded $C^{1, \alpha_{0}}$ domain. Suppose that $A \in \Lambda(\mu, \lambda, \tau)$ and $A^{*}=A$. Let $\mathcal{L}_{\varepsilon}\left(u_{\varepsilon}\right)=0$ in $B(Q, \rho) \cap \Omega$ and $\frac{\partial u_{\varepsilon}}{\partial \nu_{\varepsilon}}=g$ on $B(Q, \rho) \cap \partial \Omega$ for some $Q \in \partial \Omega$ and $0<\rho<c$. Assume that $g \in C^{\eta}(B(Q, \rho) \cap \partial \Omega)$ for some $\eta \in\left(0, \alpha_{0}\right)$. Then

$$
\left\|\nabla u_{\varepsilon}\right\|_{L^{\infty}(B(Q, \rho / 2) \cap \Omega)} \leq C\left\{\rho^{-1}\left\|u_{\varepsilon}\right\|_{L^{\infty}(B(Q, \rho) \cap \Omega)}+\|g\|_{C^{\eta}(B(Q, \rho) \cap \partial \Omega)}\right\},
$$
where $c=c(\Omega)>0$ and $C$ depends only on $d, m, \mu, \lambda, \tau, \eta$, and $\Omega$.

Let $D(\rho)=D(\rho, \psi)$ and $\Delta(\rho)=D(\rho, \psi)$ be defined by (2.7) with $\psi \in C^{1, \alpha_{0}}\left(\mathbb{R}^{d-1}\right)$, $\psi(0)=|\nabla \psi(0)|=0$, and $\|\nabla \psi\|_{C^{\alpha_{0}\left(\mathbb{R}^{d-1}\right)}} \leq M_{0}$. We will use $\|g\|_{C^{0, \eta}(K)}$ to denote

$$
\inf \left\{M:|g(x)-g(y)| \leq M|x-y|^{\beta} \text { for all } x, y \in K\right\} .
$$

Lemma 7.2. Let $0<\eta<\alpha_{0}$ and $\kappa=(1 / 4) \eta$. Let $\Phi_{\varepsilon}=\Phi_{\varepsilon}\left(x, \Omega_{\psi}, A\right)$ be defined as in Remark 6.4. There exist constants $\varepsilon_{0}>0, \theta \in(0,1)$, and $C_{0}>0$, depending only on $d, m, \mu, \lambda, \tau, \eta$, and $\left(\alpha_{0}, M_{0}\right)$, such that

$$
\left\|u_{\varepsilon}-\left\langle\Phi_{\varepsilon}, \mathbf{B}_{\varepsilon}\right\rangle\right\|_{L^{\infty}(D(\theta))} \leq \theta^{1+\kappa},
$$


for some $\mathbf{B}_{\varepsilon}=\left(b_{\varepsilon, j}^{\beta}\right) \in \mathbb{R}^{d m}$ with the property that

$$
\left|\mathbf{B}_{\varepsilon}\right| \leq C_{0} \theta^{-1}\left\|u_{\varepsilon}\right\|_{L^{\infty}(D(\theta))} \text { and }\left\langle n(0) \hat{A}, \mathbf{B}_{\varepsilon}\right\rangle=n_{i}(0) \hat{a}_{i j}^{\alpha \beta} b_{\varepsilon, j}^{\beta}=0,
$$

whenever

$$
\varepsilon<\varepsilon_{0}, \quad \mathcal{L}_{\varepsilon}\left(u_{\varepsilon}\right)=0 \text { in } D(1), \quad \frac{\partial u_{\varepsilon}}{\partial \nu_{\varepsilon}}=g \text { on } \Delta(1), \quad u_{\varepsilon}(0)=0,
$$

and

$$
\|g\|_{C^{0, \eta}(\Delta(1))} \leq 1, \quad g(0)=0, \quad\left\|u_{\varepsilon}\right\|_{L^{\infty}(D(1))} \leq 1 .
$$

Proof. Let $\mathcal{L}_{0}=-\operatorname{div}\left(A^{0} \nabla\right)$, where $A^{0}=\left(\hat{a}_{i j}^{\alpha \beta}\right)$ is a constant $m \times m$ matrix satisfying (1.2). By boundary Hölder estimates for gradients of solutions to elliptic systems with constant coefficients in $C^{1, \alpha_{0}}$ domains,

$$
\begin{aligned}
\| w- & \left\langle x,(\overline{\nabla w})_{D(r)}\right\rangle \|_{L^{\infty}(D(r))} \\
& \leq C_{1} r^{1+2 \kappa}\left\{\|g\|_{C^{\eta}(\Delta(1 / 2))}+\|w\|_{L^{\infty}(D(1 / 2))}\right\},
\end{aligned}
$$

for any $r \in(0,1 / 4)$, whenever $\mathcal{L}_{0}(w)=0$ in $D(1 / 2), \frac{\partial w}{\partial \nu_{0}}=g$ on $\Delta(1 / 2)$, and $w(0)=0$. The constant $C_{1}$ in (7.4) depends only on $d, m, \mu, \eta$, and $\left(\alpha_{0}, M_{0}\right)$. Observe that if

$$
g(0)=\left\langle n(0) A^{0},(\nabla w)(0)\right\rangle=0,
$$

then $\|g\|_{C^{\eta}(\Delta(1 / 2))} \leq C\|g\|_{C^{0, \eta}(\Delta(1 / 2))}$ and

$$
\begin{gathered}
\left|\left\langle n(0) A^{0},(\overline{\nabla w})_{D(r)}\right\rangle\right|=\left|\left\langle n(0) A^{0},(\overline{\nabla w})_{D(r)}-(\nabla w)(0)\right\rangle\right| \\
\leq C r^{2 \kappa}\left\{\|g\|_{C^{0, \eta}(\Delta(1 / 2))}+\|w\|_{L^{\infty}(D(1 / 2))}\right\} .
\end{gathered}
$$

Consequently, if we let $\mathbf{B}_{0}=\left(b_{0, j}^{\beta}\right) \in \mathbb{R}^{d m}$ with

$$
b_{0, j}^{\beta}=\left(\overline{\frac{\partial w^{\beta}}{\partial x_{j}}}\right)_{D(r)}-n_{j}(0) h^{\beta \gamma} n_{i}(0) \hat{a}_{i \ell}^{\gamma \alpha}\left(\overline{\frac{\partial w^{\alpha}}{\partial x_{\ell}}}\right)_{D(r)},
$$

where $\left(h^{\alpha \beta}\right)_{m \times m}$ is the inverse matrix of $\left(n_{i}(0) n_{j}(0) \hat{a}_{i j}^{\alpha \beta}\right)_{m \times m}$, then

$$
\left\|w-\left\langle x, \mathbf{B}_{0}\right\rangle\right\|_{L^{\infty}(D(0, r))} \leq C_{2} r^{1+2 \kappa},
$$

for any $r \in(0,1 / 4)$, provided that $\mathcal{L}_{0}(w)=0$ in $D(1 / 2), \frac{\partial w}{\partial \nu_{0}}=g$ on $\Delta(1 / 2)$, $w(0)=0$,

$$
\|g\|_{C^{0, \eta}(\Delta(1 / 2))} \leq 1, \quad g(0)=0, \quad \text { and } \quad\|w\|_{L^{\infty}(D(1 / 2))} \leq 1,
$$

where $C_{2}$ depends only on $d, m, \mu, \eta$, and $\left(\alpha_{0}, M_{0}\right)$.

Next we choose $\theta \in(0,1 / 4)$ so small that $2 C_{2} \theta^{\kappa} \leq 1$. We shall show by contradiction that for this $\theta$, there exists $\varepsilon_{0}>0$, depending only on $d, m, \mu, \lambda, \tau, \eta$, and $\left(\alpha_{0}, M_{0}\right)$, such that estimate (7.2) holds with

$$
b_{\varepsilon, j}^{\beta}=\left(\overline{\frac{\partial u_{\varepsilon}^{\beta}}{\partial x_{j}}}\right)_{D(\theta)}-n_{j}(0) h^{\beta \gamma} n_{i}(0) \hat{a}_{i \ell}^{\gamma \alpha}\left(\overline{\frac{\partial u_{\varepsilon}^{\alpha}}{\partial x_{\ell}}}\right)_{D(\theta)},
$$

if $0<\varepsilon<\varepsilon_{0}$ and $u_{\varepsilon}$ satisfies the conditions in Lemma 7.2. We recall that $\left(\hat{a}_{i j}^{\alpha \beta}\right)$ in (7.9) is the homogenized matrix given by (2.3). It is easy to verify that $n_{i}(0) \hat{a}_{i j}^{\alpha \beta} b_{\varepsilon, j}^{\beta}=0$. Also, by the divergence theorem, $\left|\mathbf{B}_{\varepsilon}\right| \leq C_{0} \theta^{-1}\left\|u_{\varepsilon}\right\|_{L^{\infty}(D(\theta))}$. 
To show (7.2) by contradiction, let's suppose that there exist sequences $\left\{\varepsilon_{k}\right\}$, $\left\{A^{k}\right\},\left\{u_{\varepsilon_{k}}\right\},\left\{g_{k}\right\}$, and $\left\{\psi_{k}\right\}$ such that $\varepsilon_{k} \rightarrow 0, A^{k} \in \Lambda(\mu, \lambda, \tau), \psi_{k}$ satisfies (2.6),

$$
\begin{aligned}
& \left\{\begin{aligned}
\mathcal{L}_{\varepsilon_{k}}^{k}\left(u_{\varepsilon_{k}}\right) & =0 & & \text { in } D_{k}(1), \\
\frac{\partial u_{\varepsilon_{k}}}{\partial \nu_{\varepsilon_{k}}} & =g_{k} & & \text { on } \Delta_{k}(1), \\
u_{\varepsilon_{k}}(0) & =g_{k}(0)=0, & &
\end{aligned}\right. \\
& \left\|g_{k}\right\|_{C^{0, \eta}\left(\Delta_{k}(1)\right)} \leq 1, \quad\left\|u_{\varepsilon_{k}}\right\|_{L^{\infty}\left(D_{k}(1)\right)} \leq 1,
\end{aligned}
$$

and

$$
\left\|u_{\varepsilon_{k}}-\left\langle\Phi_{\varepsilon_{k}}^{k}, \mathbf{B}_{\varepsilon}^{k}\right\rangle\right\|_{L^{\infty}\left(D_{k}(\theta)\right)}>\theta^{1+\kappa}
$$

where $D_{k}(r)=D\left(r, \psi_{k}\right), \Delta_{k}(r)=\Delta\left(r, \psi_{k}\right), \Phi_{\varepsilon_{k}}^{k}=\Phi_{\varepsilon_{k}}\left(x, \Omega_{\psi_{k}}, A^{k}\right)$ and $\mathbf{B}_{\varepsilon}^{k}$ is given by (7.9). By passing to subsequences we may assume that as $k \rightarrow \infty$,

$$
\begin{aligned}
\hat{A}^{k} & \rightarrow A^{0}, \\
\psi_{k} & \rightarrow \psi_{0} \quad \text { in } C^{1}\left(\left|x^{\prime}\right|<4\right), \\
g_{k}\left(x^{\prime}, \psi_{k}\left(x^{\prime}\right)\right) & \rightarrow g_{0}\left(x^{\prime}, \psi_{0}\left(x^{\prime}\right)\right) \quad \text { in } C\left(\left|x^{\prime}\right|<1\right) .
\end{aligned}
$$

Since $\left\|u_{\varepsilon_{k}}\right\|_{C^{\eta}\left(D\left(1 / 2, \psi_{k}\right)\right)}+\left\|\Phi_{\varepsilon_{k}}^{k}\right\|_{C^{\eta}\left(D\left(1 / 2, \psi_{k}\right)\right)} \leq C$ by Theorem 3.1 again by passing to subsequences, we may also assume that

$$
\begin{aligned}
& u_{\varepsilon_{k}}\left(x^{\prime}, x_{d}-\psi_{k}\left(x^{\prime}\right)\right) \rightarrow u_{0}\left(x^{\prime}, x_{d}-\psi_{0}\left(x^{\prime}\right)\right) \quad \text { uniformly on } D(1 / 2,0), \\
& R_{\varepsilon_{k}}^{k}\left(x^{\prime}, x_{d}-\psi_{k}\left(x^{\prime}\right)\right) \quad \text { converges uniformly on } D(1 / 2,0),
\end{aligned}
$$

where $R_{\varepsilon_{k}}^{k}(x)=\Phi_{\varepsilon_{k}}^{k}(x)-x$. Furthermore, in view of Theorem 2.3, we may assume that $\mathcal{L}_{0}\left(u_{0}\right)=0$ in $D\left(1 / 2, \psi_{0}\right)$ and $\frac{\partial u_{0}}{\partial \nu_{0}}=g_{0}$ on $\Delta\left(1 / 2, \psi_{0}\right)$, where $\mathcal{L}_{0}=-\operatorname{div}\left(A^{0} \nabla\right)$.

Note that by Lemma 6.3. $R_{\varepsilon_{k}}^{k}\left(x^{\prime}, x_{d}-\psi_{k}\left(x^{\prime}\right)\right)$ must converge to a constant. Since $R_{\varepsilon_{k}}^{k}(0)=0$, we deduce that $R_{\varepsilon_{k}}^{k}\left(x^{\prime}, x_{d}-\psi_{k}\left(x^{\prime}\right)\right)$ converges uniformly to 0 on $D(1 / 2,0)$. Thus, in view of (17.11)-(17.14), we may conclude that $u_{0}(0)=g(0)=0$,

$$
\|g\|_{C^{0, \eta}\left(\Delta\left(1 / 2, \psi_{0}\right)\right)} \leq 1, \quad\left\|u_{0}\right\|_{L^{\infty}\left(D\left(1 / 2, \psi_{0}\right)\right)} \leq 1,
$$

and

$$
\left\|u_{0}-\left\langle x, \mathbf{B}_{0}\right\rangle\right\|_{L^{\infty}\left(D\left(\theta, \psi_{0}\right)\right)} \geq \theta^{1+\kappa} .
$$

This, however, contradicts (7.7)-(7.8).

Remark 7.3. Let $w=\left\langle\Phi_{\varepsilon}, \mathbf{B}_{\varepsilon}\right\rangle=\Phi_{\varepsilon, j}^{\alpha \beta}(x) b_{\varepsilon, j}^{\beta}$, where $\Phi_{\varepsilon}$ and $\mathbf{B}_{\varepsilon}$ are given by Lemma 7.2. Then $\mathcal{L}_{\varepsilon}(w)=0$ and $\frac{\partial w}{\partial \nu_{\varepsilon}}=n_{i}(x) \hat{a}_{i j}^{\alpha \beta} b_{\varepsilon, j}^{\beta}$. In particular, we have $w(0)=0$ and $\frac{\partial w}{\partial \nu_{\varepsilon}}(0)=0$. Also, note that in Lemma 7.2 , one may choose any $\theta \in\left(0, \theta_{1}\right)$, where $2 C_{2} \theta_{1}^{\kappa}=1$. These observations are important to the proof of the next lemma.

Lemma 7.4. Let $\kappa, \varepsilon_{0}, \theta$ be the constants given by Lemma 7.2. Suppose that $\mathcal{L}_{\varepsilon}\left(u_{\varepsilon}\right)=0$ in $D(1, \psi), \frac{\partial u_{\varepsilon}}{\partial \nu_{\varepsilon}}=g$ on $\Delta(1, \psi)$, and $u_{\varepsilon}(0)=g(0)=0$. Assume that $\varepsilon<\theta^{\ell-1} \varepsilon_{0}$ for some $\ell \geq 1$. Then there exist $\mathbf{B}_{\varepsilon}^{j} \in \mathbb{R}^{d m}$ for $j=0,1, \ldots, \ell-1$, such that

$$
\left\langle n(0) \hat{A}, \mathbf{B}_{\varepsilon}^{j}\right\rangle=0, \quad\left|\mathbf{B}_{\varepsilon}^{j}\right| \leq C J
$$


and

$$
\left\|u_{\varepsilon}-\sum_{j=0}^{\ell-1} \theta^{\kappa j}\left\langle\Pi_{\varepsilon}^{j}, \mathbf{B}_{\varepsilon}^{j}\right\rangle\right\|_{L^{\infty}\left(D\left(\theta^{\ell}, \psi\right)\right)} \leq \theta^{\ell(1+\kappa)} J
$$

where

$$
\begin{aligned}
& \Pi_{\varepsilon}^{j}(x)=\theta^{j} \Phi_{\frac{\varepsilon}{\theta^{j}}}\left(\theta^{-j} x, \Omega_{\psi_{j}}, A\right), \\
& J=\max \left\{\|g\|_{C^{0, \eta}(\Delta(1, \psi))},\left\|u_{\varepsilon}\right\|_{L^{\infty}(D(1, \psi))}\right\},
\end{aligned}
$$

and $\psi_{j}\left(x^{\prime}\right)=\theta^{-j} \psi\left(\theta^{j} x^{\prime}\right)$.

Proof. The lemma is proved by an induction argument on $\ell$. The case $\ell=1$ follows by applying Lemma 7.2 to $u_{\varepsilon} / J$. Suppose now that Lemma 7.4 holds for some $\ell \geq 1$. Let $\varepsilon<\theta^{\ell} \varepsilon_{0}$. Consider the function

$$
w(x)=\theta^{-\ell}\left\{u_{\varepsilon}\left(\theta^{\ell} x\right)-\sum_{j=0}^{\ell-1} \theta^{\kappa j}\left\langle\Pi_{\varepsilon}^{j}\left(\theta^{\ell} x\right), \mathbf{B}_{\varepsilon}^{j}\right\rangle\right\}
$$

on $D\left(1, \psi_{\ell}\right)$. Note that $\mathcal{L}_{\frac{\varepsilon}{\theta^{\ell}}}(w)=0$ in $D\left(1, \psi_{\ell}\right), w(0)=0$, and by the induction assumption,

$$
\|w\|_{L^{\infty}\left(D\left(1, \psi_{\ell}\right)\right)} \leq \theta^{\ell \kappa} J
$$

Let

$$
h(x)=\frac{\partial w}{\partial \nu_{\frac{\varepsilon}{\theta^{\ell}}}}(x) \quad \text { on } \Delta\left(1, \psi_{\ell}\right) .
$$

Then

$$
h(x)=g\left(\theta^{\ell} x\right)-\sum_{j=1}^{\ell-1} \theta^{\kappa j}\left\langle n\left(\theta^{\ell} x\right) \hat{A}, \mathbf{B}_{\varepsilon}^{j}\right\rangle,
$$

where $n$ denotes the unit outward normal to $\Delta(1, \psi)$. It follows that $h(0)=0$. Since $\varepsilon \theta^{-\ell}<\varepsilon_{0}$, we may then apply the estimate for the case $\ell=1$ to obtain

$$
\begin{aligned}
\| w- & \left\langle\Phi_{\frac{\varepsilon}{\theta^{\ell}}}\left(x, \Omega_{\psi_{\ell}}, A\right), \mathbf{B}_{\frac{\varepsilon}{\theta^{\ell}}}\right\rangle \|_{L^{\infty}\left(D\left(\theta, \psi_{\ell}\right)\right)} \\
& \leq \theta^{1+\kappa} \max \left\{\|h\|_{C^{0, \eta}\left(\Delta\left(1, \psi_{\ell}\right)\right)},\|w\|_{L^{\infty}\left(D\left(1, \psi_{\ell}\right)\right)}\right\},
\end{aligned}
$$

where $\mathbf{B}_{\frac{\varepsilon}{\theta^{\ell}}} \in \mathbb{R}^{d m}$ satisfies the conditions $\left\langle n(0) \hat{A}, \mathbf{B}_{\frac{\varepsilon}{\theta^{\ell}}}\right\rangle=0$ and

$$
\left|\mathbf{B}_{\frac{\varepsilon}{\theta^{\ell}}}\right| \leq C \max \left\{\|h\|_{C^{0, \eta}\left(\Delta\left(1, \psi_{\ell}\right)\right)},\|w\|_{L^{\infty}\left(D\left(1, \psi_{\ell}\right)\right)}\right\} .
$$

It follows that

$$
\begin{aligned}
& \left\|u_{\varepsilon}(x)-\sum_{j=0}^{\ell-1} \theta^{\kappa j}\left\langle\Pi_{\varepsilon}^{j}(x), \mathbf{B}_{\varepsilon}^{j}\right\rangle-\theta^{\ell}\left\langle\Phi_{\frac{\varepsilon}{\theta^{\ell}}}\left(\theta^{-\ell} x, \Omega_{\psi_{\ell}}, A\right), \mathbf{B}_{\frac{\varepsilon}{\theta^{\ell}}}\right\rangle\right\|_{L^{\infty}\left(D\left(\theta^{\ell+1}, \psi\right)\right)} \\
& \leq \theta^{\ell+1+\kappa} \max \left\{\|h\|_{C^{0, \eta}\left(\Delta\left(1, \psi_{\ell}\right)\right)},\|w\|_{L^{\infty}\left(D\left(1, \psi_{\ell}\right)\right)}\right\} .
\end{aligned}
$$

To estimate the right-hand side of (7.22), we observe that

$$
\begin{aligned}
\|h\|_{C^{0, \eta}\left(\Delta\left(1, \psi_{\ell}\right)\right)} & \leq \theta^{\ell \eta}\|g\|_{C^{0, \eta}(\Delta(1, \psi))}+\sum_{j=0}^{\ell-1} \theta^{\kappa j} \cdot C J \cdot \theta^{\ell \eta}\|n\|_{C^{0, \eta}(\Delta(1, \psi))} \\
& \leq \theta^{4 \ell \kappa} J\left\{1+\frac{C\|n\|_{C^{0, \eta}(\Delta(1, \psi))}}{1-\theta^{\kappa}}\right\},
\end{aligned}
$$


since $\eta=4 \kappa$. Since $0<\eta<\alpha_{0}$, by making an initial dilation of $x$, if necessary, we may assume that $\|n\|_{C^{0, \eta}(\Delta(1, \psi))}$ is small so that

$$
\theta^{\kappa}\left\{1+\frac{C\|n\|_{C^{0, \eta}(\Delta(1, \psi))}}{1-\theta^{\kappa}}\right\} \leq 1
$$

This implies that

$$
\|h\|_{C^{0, \eta}\left(\Delta\left(1, \psi_{\ell}\right)\right)} \leq \theta^{\ell \kappa} J .
$$

This, together with (7.18) and (7.22), gives

$$
\left\|u_{\varepsilon}-\sum_{j=0}^{\ell} \theta^{\kappa j}\left\langle\Pi_{\varepsilon}^{j}, \mathbf{B}_{\varepsilon}^{j}\right\rangle\right\|_{L^{\infty}\left(D\left(\theta^{\ell+1}, \psi\right)\right)} \leq \theta^{(\ell+1)(1+\kappa)} J
$$

where we have chosen $\mathbf{B}_{\varepsilon}^{\ell}=\theta^{-\ell \kappa} \mathbf{B}_{\frac{\varepsilon}{\theta^{\ell}}}$. Finally, in view of (7.21), (7.18), and (7.24), we have $\left|\mathbf{B}_{\varepsilon}^{\ell}\right| \leq C J$. This completes the induction argument.

Lemma 7.5. Suppose that $\mathcal{L}_{\varepsilon}\left(u_{\varepsilon}\right)=0$ in $D(1)$ and $\frac{\partial u_{\varepsilon}}{\partial \nu_{\varepsilon}}=g$ on $\Delta(1)$. Then

$$
\int_{D(\rho)}\left|\nabla u_{\varepsilon}\right|^{2} d x \leq C \rho^{d}\left\{\left\|u_{\varepsilon}\right\|_{L^{\infty}(D(1))}^{2}+\|g\|_{C^{\eta}(\Delta(1))}^{2}\right\}
$$

for any $0<\rho<(1 / 2)$, where $C$ depends only on $\mu, \lambda, \tau, \eta$, and $\left(M_{0}, \alpha_{0}\right)$.

Proof. By subtracting a constant we may assume that $u_{\varepsilon}(0)=0$. We may also assume that $g(0)=0$. To see this, consider

$$
v_{\varepsilon}^{\alpha}(x)=u_{\varepsilon}^{\alpha}(x)-\Phi_{\varepsilon, j}^{\alpha \beta}(x) n_{j}(0) b^{\beta},
$$

where $\left(b^{\beta}\right) \in \mathbb{R}^{m}$ solves the linear system $n_{i}(0) n_{j}(0) \hat{a}_{i j}^{\alpha \beta} b^{\beta}=g^{\alpha}(0)$. Then $\mathcal{L}_{\varepsilon}\left(v_{\varepsilon}\right)=$ 0 in $D(1), v_{\varepsilon}(0)=0$, and

$$
\left(\frac{\partial v_{\varepsilon}}{\partial \nu_{\varepsilon}}\right)^{\alpha}(x)=g^{\alpha}(x)-n_{i}(x) \hat{a}_{i j}^{\alpha \beta} n_{j}(0) b^{\beta} \quad \text { on } \Delta(1) .
$$

Thus $\frac{\partial v_{\varepsilon}}{\partial \nu_{\varepsilon}}(0)=0$. Since $\left\|\Phi_{\varepsilon}\right\|_{L^{\infty}(D(1))}+\left\|\nabla \Phi_{\varepsilon}\right\|_{L^{\infty}(D(1))} \leq C$, the desired estimate for $u_{\varepsilon}$ follows from the corresponding estimate for $v_{\varepsilon}$.

Under the assumption that $u_{\varepsilon}(0)=g(0)=0$, we will show that

$$
\left\|u_{\varepsilon}\right\|_{L^{\infty}(D(\rho))} \leq C \rho\left\{\left\|u_{\varepsilon}\right\|_{L^{\infty}(D(1))}+\|g\|_{C^{\eta}(\Delta(1))}\right\},
$$

for any $0<\rho<(1 / 2)$. Estimate (17.26) follows from (7.27) by Cacciopoli's inequality (3.4).

Let $\kappa, \varepsilon_{0}, \theta$ be the constants given by Lemma 7.2 . Let $0<\varepsilon<\theta \varepsilon_{0}$ (the case $\varepsilon \geq \theta \varepsilon_{0}$ follows from the classical regularity estimates). Suppose that

$$
\theta^{i+1} \leq \frac{\varepsilon}{\varepsilon_{0}}<\theta^{i} \quad \text { for some } i \geq 1
$$


Let $\rho \in(0,1 / 2)$. We first consider the case $\frac{\varepsilon}{\varepsilon_{0}} \leq \rho<\theta$. Then $\theta^{\ell+1} \leq \rho<\rho^{\ell}$ for some $\ell=1, \ldots, i$. It follows that

$$
\begin{aligned}
& \left\|u_{\varepsilon}\right\|_{L^{\infty}(D(\rho))} \leq\left\|u_{\varepsilon}\right\|_{L^{\infty}\left(D\left(\theta^{\ell}\right)\right)} \\
& \leq\left\|u_{\varepsilon}-\sum_{j=0}^{\ell-1} \theta^{\kappa j}\left\langle\Pi_{\varepsilon}^{j}, \mathbf{B}_{\varepsilon}^{j}\right\rangle\right\|_{L^{\infty}\left(D\left(\theta^{\ell}\right)\right)}+\sum_{j=0}^{\ell-1} \theta^{\kappa j}\left|\mathbf{B}_{\varepsilon}^{j}\right|\left\|\Pi_{\varepsilon}^{j}\right\|_{L^{\infty}\left(D\left(\theta^{\ell}\right)\right)} \\
& \leq \theta^{\ell(1+\kappa)} J+C J \sum_{j=0}^{\ell-1} \theta^{\kappa j}\left\|\Pi_{\varepsilon}^{j}\right\|_{L^{\infty}\left(D\left(\theta^{\ell}\right)\right)},
\end{aligned}
$$

where $J=\max \left\{\|g\|_{C^{0, \eta}(D(1))},\left\|u_{\varepsilon}\right\|_{L^{\infty}(D(1))}\right\}$ and we have used Lemma 7.4. Recall that $\Pi_{\varepsilon}^{j}(x)=\theta^{j} \Phi_{\frac{\varepsilon}{\theta^{j}}}\left(\theta^{-j} x, \Omega_{\psi_{j}}, A\right)$. By Remark 6.4 we have $\Pi_{\varepsilon}^{j}(0)=0$ and $\left\|\nabla \Pi_{\varepsilon}^{j}\right\|_{L^{\infty}(D(1))} \leq C$. Hence,

$$
\left\|\Pi_{\varepsilon}^{j}\right\|_{L^{\infty}\left(D\left(\theta^{\ell}\right)\right)} \leq C \theta^{\ell} .
$$

This, together with (7.28) , gives $\left\|u_{\varepsilon}\right\|_{L^{\infty}(D(\rho))} \leq C \rho J$ for any $\frac{\varepsilon}{\varepsilon_{0}} \leq \rho<\frac{1}{2}$ (the case $\theta \leq \rho<(1 / 2)$ is trivial $)$.

To treat the case $0<\rho<\frac{\varepsilon}{\varepsilon_{0}}$, we use a blow-up argument. Let $w(x)=\varepsilon^{-1} u_{\varepsilon}(\varepsilon x)$. Then $\mathcal{L}_{1}(w)=0$ in $D\left(2 \varepsilon_{0}^{-1}, \psi_{\varepsilon}\right)$ and $\frac{\partial w}{\partial \nu_{1}}(x)=g(\varepsilon x)$ on $\Delta\left(2 \varepsilon_{0}^{-1}, \psi_{\varepsilon}\right)$, where $\psi_{\varepsilon}\left(x^{\prime}\right)=$ $\varepsilon^{-1} \psi\left(\varepsilon x^{\prime}\right)$. By the classical regularity estimate,

$$
\|\nabla w\|_{L^{\infty}\left(D\left(\frac{1}{\varepsilon_{0}}, \psi_{\varepsilon}\right)\right)} \leq C\left\{\|w\|_{L^{\infty}\left(D\left(\frac{2}{\varepsilon_{0}}, \psi_{\varepsilon}\right)\right)}+\left\|\frac{\partial w}{\partial \nu_{1}}\right\|_{C^{\eta}\left(\Delta\left(\frac{2}{\varepsilon_{0}}, \psi_{\varepsilon}\right)\right)}\right\} .
$$

It follows that

$$
\left\|\nabla u_{\varepsilon}\right\|_{L^{\infty}\left(D\left(\frac{\varepsilon}{\varepsilon_{0}}\right)\right)} \leq C\left\{\varepsilon^{-1}\|u\|_{L^{\infty}\left(D\left(\frac{2 \varepsilon}{\varepsilon_{0}}\right)\right)}+\|g\|_{C^{\eta}(\Delta(1))}\right\} \leq C J,
$$

where we have used the estimate (7.27) with $\rho=\frac{2 \varepsilon}{\varepsilon_{0}}$ for the last inequality. Finally, since $u_{\varepsilon}(0)=0$, for $0<\rho<\frac{\varepsilon}{\varepsilon_{0}}$, we obtain

$$
\left\|u_{\varepsilon}\right\|_{L^{\infty}(D(\rho))} \leq C \rho\left\|\nabla u_{\varepsilon}\right\|_{L^{\infty}\left(D\left(\frac{\varepsilon}{\varepsilon_{0}}\right)\right)} \leq C \rho J .
$$

This completes the proof of (7.27).

Proof of Theorem 7.1. By rescaling we may assume that $\rho=1$. By a change of the coordinate system, we may deduce from Lemma 7.5 that if $P \in \partial \Omega,|P-Q|<\frac{1}{2}$, and $0<r<\frac{1}{4}$,

$$
\int_{B(P, r) \cap \Omega}\left|\nabla u_{\varepsilon}\right|^{2} d x \leq C r^{d}\left\{\left\|u_{\varepsilon}\right\|_{L^{\infty}(B(Q, 1) \cap \Omega)}^{2}+\|g\|_{C^{\eta}(B(Q, 1) \cap \partial \Omega)}^{2}\right\},
$$

where $C$ depends only on $d, m, \mu, \lambda, \tau, \eta$, and $\Omega$. This, together with the interior estimate (2.16), implies that

$$
\left\|\nabla u_{\varepsilon}\right\|_{L^{\infty}\left(B\left(Q, \frac{1}{2}\right) \cap \Omega\right)} \leq C\left\{\left\|u_{\varepsilon}\right\|_{L^{\infty}(B(Q, 1) \cap \Omega)}+\|g\|_{C^{\eta}(B(Q, 1) \cap \partial \Omega)}\right\} .
$$

The proof of Theorem 7.1 is now complete. 


\section{Proof of Theorem 1.2}

Under the condition $A \in \Lambda(\lambda, \mu, \tau)$, we have proved in Section 5 that

$$
\left|N_{\varepsilon}(x, y)\right| \leq \frac{C}{|x-y|^{d-2}} \quad \text { if } d \geq 3 .
$$

With the additional assumption $A^{*}=A$, we may use Theorem 7.1 to show that for $d \geq 3$,

$$
\begin{aligned}
\left|\nabla_{x} N_{\varepsilon}(x, y)\right|+\left|\nabla_{y} N_{\varepsilon}(x, y)\right| & \leq \frac{C}{|x-y|^{d-1}}, \\
\left|\nabla_{x} \nabla_{y} N_{\varepsilon}(x, y)\right| & \leq \frac{C}{|x-y|^{d}} .
\end{aligned}
$$

If $d=2$, one obtains $\left|N_{\varepsilon}(x, y)\right| \leq C_{\gamma}|x-y|^{-\gamma}$ and $\left|\nabla_{x} N_{\varepsilon}(x, y)\right|+\left|\nabla_{y} N_{\varepsilon}(x, y)\right| \leq$ $C_{\gamma}|x-y|^{-1-\gamma}$ for any $\gamma>0$ (this is not sharp, but sufficient for the proof of Theorem 1.2). Now, given $F \in L^{q}(\Omega)$ for some $q>d$, let

$$
v_{\varepsilon}(x)=\int_{\Omega} N_{\varepsilon}(x, y) F(y) d y .
$$

Then $\mathcal{L}_{\varepsilon}\left(v_{\varepsilon}\right)=F$ in $\Omega$ and $\frac{\partial v_{\varepsilon}}{\partial \nu_{\varepsilon}}=-\frac{1}{|\partial \Omega|} \int_{\Omega} F$ on $\partial \Omega$. Furthermore, it follows from pointwise estimates on $\left|\nabla_{x} N_{\varepsilon}(x, y)\right|$ that $\left\|\nabla v_{\varepsilon}\right\|_{L^{\infty}(\Omega)} \leq C\|F\|_{L^{q}(\Omega)}$. Thus, by subtracting $v_{\varepsilon}$ from $u_{\varepsilon}$, we may assume that $F=0$ in Theorem 1.2. In this case we may deduce from Theorems 7.1 and 3.1 that for $Q \in \partial \Omega$,

$$
\left\|\nabla u_{\varepsilon}\right\|_{L^{\infty}(B(Q, \rho / 2) \cap \Omega)} \leq C\left\{\left(f_{B(Q, \rho) \cap \Omega}\left|\nabla u_{\varepsilon}\right|^{2}\right)^{1 / 2}+\|g\|_{C^{\eta}(\Delta(Q, \rho))}\right\},
$$

where $C$ depends only on $d, m, \mu, \lambda, \tau, \eta$, and $\Omega$. Since $\left\|\nabla u_{\varepsilon}\right\|_{L^{2}(\Omega)} \leq C\|g\|_{L^{2}(\partial \Omega)}$, the estimate $\left\|\nabla u_{\varepsilon}\right\|_{L^{\infty}(\Omega)} \leq C\|g\|_{C^{\eta}(\partial \Omega)}$ follows from (8.3) and the interior estimate (2.16) by a covering argument.

\section{Proof of Theorem 1.3}

As we mentioned in Section 1, the case $p=2$ is proved in 23] (for Lipschitz domains). To handle the case $p>2$, we need the following weak reverse Hölder inequality.

Lemma 9.1. Let $\Omega$ be a bounded $C^{1, \alpha_{0}}$ domain. Suppose that $A \in \Lambda(\lambda, \mu, \tau)$ and $A^{*}=A$. Then, for $Q \in \partial \Omega$ and $0<r<r_{0}$,

$$
\sup _{B(Q, r) \cap \partial \Omega}\left(\nabla u_{\varepsilon}\right)^{*} \leq C\left\{f_{B(Q, 2 r) \cap \partial \Omega}\left|\left(\nabla u_{\varepsilon}\right)^{*}\right|^{2} d \sigma\right\}^{1 / 2},
$$

where $u_{\varepsilon} \in W^{1,2}(B(Q, 3 r) \cap \Omega)$ is a weak solution to $\mathcal{L}_{\varepsilon}\left(u_{\varepsilon}\right)=0$ in $B(Q, 3 r) \cap \Omega$ with either $\frac{\partial u_{\varepsilon}}{\partial \nu_{\varepsilon}}=0$ or $u_{\varepsilon}=0$ on $B(Q, 3 r) \cap \partial \Omega$.

Proof. Recall that the nontangential maximal function of $\left(\nabla u_{\varepsilon}\right)^{*}$ is defined by

$$
\left(\nabla u_{\varepsilon}\right)^{*}(P)=\sup \left\{\left|\nabla u_{\varepsilon}(x)\right|: x \in \Omega \text { and }|x-P|<C_{0} \operatorname{dist}(x, \partial \Omega)\right\},
$$

for $P \in \partial \Omega$, where $C_{0}=C(\Omega)>1$ is sufficiently large. Note that

$$
\left(\nabla u_{\varepsilon}\right)^{*}(P)=\max \left\{\mathcal{M}_{r, 1}\left(\nabla u_{\varepsilon}\right), \mathcal{M}_{r, 2}\left(\nabla u_{\varepsilon}\right)\right\},
$$


where

$$
\begin{gathered}
\mathcal{M}_{r, 1}\left(\nabla u_{\varepsilon}\right)(P)=\sup \left\{\left|\nabla u_{\varepsilon}(x)\right|: x \in \Omega,|x-P| \leq c_{0} r,\right. \\
\left.\quad \text { and }|x-P|<C_{0} \operatorname{dist}(x, \partial \Omega)\right\}, \\
\mathcal{M}_{r, 2}\left(\nabla u_{\varepsilon}\right)(P)=\sup \left\{\left|\nabla u_{\varepsilon}(x)\right|: x \in \Omega,|x-P|>c_{0} r,\right. \\
\left.\quad \text { and }|x-P|<C_{0} \operatorname{dist}(x, \partial \Omega)\right\},
\end{gathered}
$$

and $c_{0}=c(\Omega)>0$ is sufficiently small. Using interior estimate (2.16), it is easy to see that $\sup _{B(Q, r) \cap \partial \Omega} \mathcal{M}_{r, 2}\left(\nabla u_{\varepsilon}\right)$ is bounded by the right-hand side of (9.1). To estimate $\mathcal{M}_{r, 1}\left(\nabla u_{\varepsilon}\right)$, we observe that

$$
\begin{aligned}
\sup _{B(Q, r) \cap \partial \Omega} \mathcal{M}_{r, 1}\left(\nabla u_{\varepsilon}\right) & \leq \sup _{B(Q, 3 r / 2) \cap \Omega}\left|\nabla u_{\varepsilon}\right| \\
& \leq C\left\{f_{B(Q, 2 r) \cap \Omega}\left|\nabla u_{\varepsilon}\right|^{2} d x\right\}^{1 / 2} \\
& \leq C\left\{f_{B(Q, 2 r) \cap \partial \Omega}\left|\left(\nabla u_{\varepsilon}\right)^{*}\right|^{2} d \sigma\right\}^{1 / 2} .
\end{aligned}
$$

We point out that the second inequality in (9.2) follows from the boundary Lipschitz estimate. For the Neumann condition $\frac{\partial u_{\varepsilon}}{\partial \nu_{\varepsilon}}=0$ on $B(Q, 3 r) \cap \Omega$, the estimate was given by Theorem 7.1, while the case of the Dirichlet condition follows from Theorem 2 in [3, p. 805].

Lemma 9.2. Suppose that $A \in \Lambda(\lambda, \mu, \tau)$ and $A^{*}=A$. Let $p>2$ and let $\Omega$ be a bounded Lipschitz domain. Assume that

$$
\left(f_{B(Q, r) \cap \partial \Omega}\left|\left(\nabla u_{\varepsilon}\right)^{*}\right|^{p} d \sigma\right)^{1 / p} \leq C\left(f_{B(Q, 2 r) \cap \partial \Omega}\left|\left(\nabla u_{\varepsilon}\right)^{*}\right|^{2} d \sigma\right)^{1 / 2},
$$

whenever $u_{\varepsilon} \in W^{1,2}(B(Q, 3 r) \cap \Omega)$ is a weak solution to $\mathcal{L}_{\varepsilon}\left(u_{\varepsilon}\right)=0$ in $B(Q, 3 r) \cap \Omega$ and $\frac{\partial u_{\varepsilon}}{\partial \nu_{\varepsilon}}=0$ on $B(Q, 3 r) \cap \partial \Omega$ for some $Q \in \partial \Omega$ and $0<r<r_{0}$. Then the weak solutions to $\mathcal{L}_{\varepsilon}\left(u_{\varepsilon}\right)=0$ in $\Omega$ and $\frac{\partial u_{\varepsilon}}{\partial \nu_{\varepsilon}}=g \in L^{p}(\partial \Omega)$ satisfy the estimate $\left\|\left(\nabla u_{\varepsilon}\right)^{*}\right\|_{L^{p}(\partial \Omega)} \leq C\|g\|_{L^{p}(\partial \Omega)}$.

Proof. This follows by a real variable argument originating in [9] and further developed in [29]-31]. In [25] the argument was used to prove that for any given $p>2$ and Lipschitz domain $\Omega$, the solvability of the Neumann problem for Laplace's equation $\Delta u=0$ in $\Omega$ with $L^{p}$ boundary data is equivalent to a weak reverse Hölder inequality, similar to (9.3). With the solvability of the $L^{2}$ Neumann problem for $\mathcal{L}_{\varepsilon}\left(u_{\varepsilon}\right)=0$ (23]) and interior estimate (2.16), the proof of the sufficiency of the weak reverse Hölder inequality in [25, pp. 1819-1821] extends directly to the present case. We omit the details.

It follows from Lemmas 9.1 and 9.2 that Theorem 1.3 holds for $p>2$. To handle the case $1<p<2$, as in the case of Laplace's equation [12, one considers the solutions of the $L^{2}$ Neumann problem with atomic data $\frac{\partial u_{\varepsilon}}{\partial \nu_{\varepsilon}}=a$, where $\int_{\partial \Omega} a=0$, 
$\operatorname{supp}(a) \subset B(Q, r) \cap \partial \Omega$ for some $Q \in \partial \Omega$ and $0<r<r_{0}$, and $\|a\|_{L^{\infty}(\partial \Omega)} \leq r^{1-d}$. One needs to show that

$$
\int_{\partial \Omega}\left(\nabla u_{\varepsilon}\right)^{*} d \sigma \leq C
$$

The case $1<p<2$ follows from (9.4) by interpolation.

To prove (9.4), one first uses the Hölder inequality and the $L^{2}$ estimate

$$
\left\|\left(\nabla u_{\varepsilon}\right)^{*}\right\|_{L^{2}(\partial \Omega)} \leq C\|a\|_{L^{2}(\partial \Omega)} \leq C r^{\frac{1-d}{2}}
$$

to see that

$$
\int_{B(Q, C r) \cap \partial \Omega}\left(\nabla u_{\varepsilon}\right)^{*} d \sigma \leq C .
$$

Next, to estimate $(\nabla u)^{*}$ on $\partial \Omega \backslash B(Q, C r)$, we show that

$$
\int_{B\left(P_{0}, c \rho\right) \cap \partial \Omega}\left(\nabla u_{\varepsilon}\right)^{*} d \sigma \leq C\left(\frac{r}{\rho}\right)^{\gamma},
$$

for some $\gamma>0$, where $\rho=\left|P_{0}-Q\right| \geq C r$. Note that

$$
u_{\varepsilon}(x)=b+\int_{B(Q, r) \cap \partial \Omega}\left\{N_{\varepsilon}(x, y)-N_{\varepsilon}(x, Q)\right\} a(y) d \sigma(y)
$$

for some $b \in \mathbb{R}^{m}$. It follows that

$$
\left|\nabla u_{\varepsilon}(x)\right| \leq C f_{B(Q, r) \cap \partial \Omega}\left|\nabla_{x}\left\{N_{\varepsilon}(x, y)-N_{\varepsilon}(x, Q)\right\}\right| d \sigma(y) .
$$

Hence, if $z \in \Omega$ and $c \rho \leq|z-P|<C_{0} \delta(z)$ for some $P \in B\left(P_{0}, c \rho\right) \cap \partial \Omega$,

$$
\begin{aligned}
\left|\nabla u_{\varepsilon}(z)\right| & \leq C\left(f_{B(z, c \delta(z))}|\nabla u(x)|^{2} d x\right)^{1 / 2} \\
& \leq C f_{B(Q, r) \cap \partial \Omega}\left(f_{B(z, c \delta(z))}\left|\nabla_{x}\left\{N_{\varepsilon}(x, y)-N_{\varepsilon}(x, Q)\right\}\right|^{2} d x\right)^{1 / 2} d \sigma(y) \\
& \leq C \rho^{1-d}\left(\frac{r}{\rho}\right)^{\gamma},
\end{aligned}
$$

where $\delta(z)=\operatorname{dist}(z, \partial \Omega)$ and we have used the interior estimate, Minkowski's inequality, and Theorem 5.2. This implies that

$$
\int_{B\left(P_{0}, c \rho\right) \cap \partial \Omega} \mathcal{M}_{2, \rho}\left(\nabla u_{\varepsilon}\right) d \sigma \leq C\left(\frac{r}{\rho}\right)^{\gamma} .
$$

Finally, to estimate $\mathcal{M}_{1, \rho}\left(\nabla u_{\varepsilon}\right)$, we note that the $L^{2}$ nontangential maximal function estimate, together with an integration argument, gives

$$
\int_{B\left(P_{0}, c \rho\right) \cap \partial \Omega}\left|\mathcal{M}_{1, \rho}\left(\nabla u_{\varepsilon}\right)\right|^{2} d \sigma \leq \frac{C}{\rho} \int_{B\left(P_{0}, 2 c \rho\right) \cap \Omega}\left|\nabla u_{\varepsilon}\right|^{2} d x
$$

(see [12] for the case of Laplace's equation). It follows by Hölder inequality that

$$
\begin{aligned}
\int_{B\left(P_{0}, c \rho\right) \cap \partial \Omega} \mathcal{M}_{1, \rho}\left(\nabla u_{\varepsilon}\right) d \sigma & \leq C \rho^{d-1}\left(f_{B\left(P_{0}, 2 c \rho\right) \cap \Omega}\left|\nabla u_{\varepsilon}\right|^{2} d x\right)^{1 / 2} \\
& \leq C\left(\frac{r}{\rho}\right)^{\gamma}
\end{aligned}
$$


where the last inequality follows from (9.8) and Theorem 5.2, In view of (9.9) and (9.11), we have proved (9.5). The desired estimate

$$
\int_{\partial \Omega \backslash B(Q, C r)}\left(\nabla u_{\varepsilon}\right)^{*} d \sigma \leq C
$$

follows from (9.5) by a simple covering argument. This completes the proof of (9.4) and hence of Theorem 1.3 .

Remark 9.3. The estimate $\left\|\nabla u_{\varepsilon}\right\|_{L^{q}(\Omega)} \leq C\|g\|_{L^{p}(\partial \Omega)}$ with $q=\frac{p d}{d-1}$ in Theorem 1.3 follows from Theorem 1.1 using the fact that $L^{p}(\partial \Omega) \subset B^{-\frac{1}{q}, q}(\partial \Omega)$. The estimate also follows from the observation that $\|w\|_{L^{q}(\Omega)} \leq C\left\|(w)^{*}\right\|_{L^{p}(\partial \Omega)}$ for any $w$ in a Lipschitz domain $\Omega$. To see this, we note that

$$
|w(x)| \leq C \int_{\partial \Omega} \frac{(w)^{*}(Q)}{|x-Q|^{d-1}} d \sigma(Q) .
$$

By a duality argument, it then suffices to show that the operator

$$
I_{1}(f)(x)=\int_{\Omega} \frac{f(y)}{|x-y|^{d-1}} d y
$$

is bounded from $L^{q^{\prime}}(\Omega)$ to $L^{p^{\prime}}(\partial \Omega)$. This may be proved by using fractional and singular integral estimates (see e.g. [30, p. 712]).

Remark 9.4. Suppose that $d \geq 3$. For $g \in L^{p}(\partial \Omega)$, consider the $L^{p}$ Neumann problem in the exterior domain $\Omega_{-}=\mathbb{R}^{d} \backslash \bar{\Omega}$,

$$
\left\{\begin{aligned}
\mathcal{L}_{\varepsilon}\left(u_{\varepsilon}\right) & =0 \quad \text { in } \Omega_{-}, \\
\frac{\partial u_{\varepsilon}}{\partial \nu_{\varepsilon}} & =g \quad \text { on } \partial \Omega, \\
\left(\nabla u_{\varepsilon}\right)^{*} & \in L^{p}(\partial \Omega) \text { and } u_{\varepsilon}(x)=O\left(|x|^{2-d}\right) \quad \text { as }|x| \rightarrow \infty .
\end{aligned}\right.
$$

It follows from [23] that if $p=2$ and $\Omega$ is a bounded Lipschitz domain with connected boundary, the unique solution to (9.13) satisfies the estimate $\left\|\left(\nabla u_{\varepsilon}\right)^{*}\right\|_{L^{2}(\partial \Omega)}$ $\leq C\|g\|_{L^{2}(\partial \Omega)}$ (if $\partial \Omega$ is not connected, the data $g$ needs to satisfy some compatibility conditions). A careful inspection of Theorem 1.3 shows that the $L^{2}$ results extend to $L^{p}$ for $1<p<\infty$, if $\Omega$ is a bounded $C^{1, \alpha}$ domain.

\section{0. $L^{p}$ Regularity PRoblem}

In this section we outline the proof of the following.

Theorem 10.1. Suppose that $A \in \Lambda(\mu, \lambda, \tau)$ and $A^{*}=A$. Let $\Omega$ be a bounded $C^{1, \alpha}$ domain with connected boundary and let $1<p<\infty$. Then, for any $f \in W^{1, p}(\partial \Omega)$, the unique solution to $\mathcal{L}_{\varepsilon}\left(u_{\varepsilon}\right)=0$ in $\Omega, u_{\varepsilon}=f$ on $\partial \Omega$, and $\left(\nabla u_{\varepsilon}\right)^{*} \in L^{p}(\partial \Omega)$ satisfies the estimate

$$
\left\|\left(\nabla u_{\varepsilon}\right)^{*}\right\|_{L^{p}(\partial \Omega)} \leq C\left\|\nabla_{t a n} f\right\|_{L^{p}(\partial \Omega)},
$$

where $C$ depends only on $d, m, p, \mu, \lambda, \tau$, and $\Omega$.

The case $p=2$ was proved in [23] for Lipschitz domains. The case $p>2$ follows from Lemma 9.1 and the following analog of Lemma 9.2 
Lemma 10.2. Suppose that $A \in \Lambda(\lambda, \mu, \tau)$ and $A^{*}=A$. Let $p>2$ and let $\Omega$ be a bounded Lipschitz domain with connected boundary. Assume that

$$
\left(f_{B(Q, r) \cap \partial \Omega}\left|\left(\nabla u_{\varepsilon}\right)^{*}\right|^{p} d \sigma\right)^{1 / p} \leq C_{0}\left(f_{B(Q, 2 r) \cap \partial \Omega}\left|\left(\nabla u_{\varepsilon}\right)^{*}\right|^{2} d \sigma\right)^{1 / 2}
$$

whenever $u_{\varepsilon} \in W^{1,2}(B(Q, 3 r) \cap \Omega)$ is a weak solution to $\mathcal{L}_{\varepsilon}\left(u_{\varepsilon}\right)=0$ in $B(Q, 3 r) \cap \Omega$ and $u_{\varepsilon}=0$ on $B(Q, 3 r) \cap \partial \Omega$ for some $Q \in \partial \Omega$ and $0<r<r_{0}$. Then the weak solution to $\mathcal{L}_{\varepsilon}\left(u_{\varepsilon}\right)=0$ in $\Omega$ and $u_{\varepsilon}=f \in W^{1, p}(\partial \Omega)$ satisfies the estimate $\left\|\left(\nabla u_{\varepsilon}\right)^{*}\right\|_{L^{p}(\partial \Omega)} \leq C\left\|\nabla_{\text {tan }} f\right\|_{L^{p}(\partial \Omega)}$, where $C$ depends only on $d, m, p, \mu, \lambda, \tau, r_{0}$, $C_{0}$, and $\Omega$.

The proof of Lemma 10.2 is similar to that of Lemma 9.2. We refer the reader to 24] where a similar statement was proved for elliptic equations with constant coefficients.

To handle the case $1<p<2$, we follow the approach for Laplace's equation in Lipschitz domains [12] and consider $L^{2}$ solutions with Dirichlet data $u_{\varepsilon}=a$, where $\operatorname{supp}(a) \subset B(Q, r) \cap \partial \Omega$ for some $Q \in \partial \Omega$ and $0<r<r_{0}$, and $\left\|\nabla_{\text {tan }} a\right\|_{L^{\infty}(\partial \Omega)} \leq$ $r^{1-d}$. By interpolation it suffices to show estimate (9.4). Note that $|a| \leq C r^{2-d}$. Using the estimates on Green's functions in [3], one has

$$
\left|\nabla u_{\varepsilon}(x)\right| \leq \frac{C r}{|x-Q|^{d}} \quad \text { if }|x-Q| \geq C r .
$$

Estimate (9.4) follows easily from the $L^{2}$ estimate $\left\|\left(\nabla u_{\varepsilon}\right)^{*}\right\|_{L^{2}(\partial \Omega)} \leq C\left\|\nabla_{t a n} a\right\|_{L^{2}(\partial \Omega)}$ and (10.3).

Remark 10.3. One may also consider the $L^{p}$ regularity problem for the exterior domain: given $f \in W^{1, p}(\partial \Omega)$, find a solution $u_{\varepsilon}$ to $\mathcal{L}_{\varepsilon}\left(u_{\varepsilon}\right)=0$ in $\Omega_{-}$such that $u_{\varepsilon}=f$ on $\partial \Omega,\left(\nabla u_{\varepsilon}\right)^{*} \in L^{p}(\partial \Omega)$, and $u_{\varepsilon}(x)=O\left(|x|^{2-d}\right)$ as $|x| \rightarrow \infty$. It follows from [23] that if $\Omega$ is a bounded Lipschitz domain in $\mathbb{R}^{d}, d \geq 3$, then the unique solution to the $L^{2}$ regularity problem in $\Omega_{-}$satisfies the estimate $\left\|\left(\nabla u_{\varepsilon}\right)^{*}\right\|_{L^{2}(\partial \Omega)} \leq$ $C\left\|\nabla_{\text {tan }} f\right\|_{W^{1,2}(\partial \Omega)}$. An inspection of Theorem 10.1 shows that the $L^{2}$ result extends to $L^{p}$ for $1<p<\infty$, if $\Omega$ is a $C^{1, \alpha}$ domain.

\section{REPRESENTATION BY LAYER POTENTIALS}

For $f \in L^{p}(\partial \Omega)$, the single layer potential $u_{\varepsilon}=\mathcal{S}_{\varepsilon}(f)$ and double layer potential $w_{\varepsilon}=\mathcal{D}_{\varepsilon}(f)$ for the operator $\mathcal{L}_{\varepsilon}$ in $\Omega$ are defined by

$$
\begin{aligned}
u_{\varepsilon}^{\alpha}(x) & =\int_{\partial \Omega} \Gamma_{A, \varepsilon}^{\alpha \beta}(x, y) f^{\beta}(y) d \sigma(y), \\
w_{\varepsilon}^{\alpha}(x) & =\int_{\partial \Omega}\left(\frac{\partial}{\partial \nu_{\varepsilon}^{*}}\left\{\Gamma_{A^{*}, \varepsilon}^{\alpha}(y, x)\right\}\right)^{\beta} f^{\beta}(y) d \sigma(y),
\end{aligned}
$$

where $\Gamma_{A, \varepsilon}(x, y)$ and $\Gamma_{A^{*}, \varepsilon}(x, y)=\left(\Gamma_{A, \varepsilon}(y, x)\right)^{*}$ are the fundamental solutions for $\mathcal{L}_{\varepsilon}$ and $\left(\mathcal{L}_{\varepsilon}\right)^{*}$, respectively. Both $\mathcal{S}_{\varepsilon}(f)$ and $\mathcal{D}_{\varepsilon}(f)$ are solutions of $\mathcal{L}_{\varepsilon}(u)=0$ in $\mathbb{R}^{d} \backslash \partial \Omega$. Under the assumptions that $A \in \Lambda(\mu, \lambda, \tau)$ and $\Omega$ is a bounded Lipschitz domain, it was proved in 23 that for $1<p<\infty$,

$$
\left\|\left(\nabla \mathcal{S}_{\varepsilon}(f)\right)^{*}\right\|_{L^{p}(\Omega)}+\left\|\left(\mathcal{D}_{\varepsilon}(f)\right)^{*}\right\|_{L^{p}(\partial \Omega)} \leq C_{p}\|f\|_{L^{p}(\partial \Omega)},
$$

where $C_{p}$ depends only on $d, m, \mu, \lambda, \tau, p$, and the Lipschitz character of $\Omega$. Furthermore, $\left(\nabla u_{\varepsilon}\right)_{ \pm}(P)$ exists for a.e. $P \in \partial \Omega,\left(\frac{\partial u_{\varepsilon}}{\partial \nu_{\varepsilon}}\right)_{ \pm}=\left( \pm \frac{1}{2} I+\mathcal{K}_{A, \varepsilon}\right)(f)$, and 
$\left(w_{\varepsilon}\right)_{ \pm}=\left(\mp \frac{1}{2} I+\mathcal{K}_{A^{*}, \varepsilon}^{*}\right)(f)$, where $\mathcal{K}_{A^{*}, \varepsilon}$ is the adjoint of $\mathcal{K}_{A^{*}, \varepsilon}$. Here $(u)_{ \pm}$denotes the nontangential limits on $\partial \Omega$ of $u$, taken from $\Omega$ and $\Omega_{-}$, respectively.

Let $L_{0}^{p}\left(\partial \Omega, \mathbb{R}^{m}\right)$ denote the space of functions in $L^{p}\left(\partial \Omega, \mathbb{R}^{m}\right)$ with mean value zero.

Theorem 11.1. Let $\Omega$ be a bounded $C^{1, \alpha}$ domain in $\mathbb{R}^{d}, d \geq 3$, with connected boundary. Suppose that $A \in \Lambda(\mu, \lambda, \tau)$ and $A^{*}=A$. Then, for $1<p<\infty$,

$$
\begin{aligned}
\frac{1}{2} I+\mathcal{K}_{A, \varepsilon} & : L_{0}^{p}\left(\partial \Omega, \mathbb{R}^{m}\right) \\
-\frac{1}{2} I+\mathcal{K}_{A^{*}, \varepsilon}^{*}: L^{p}\left(\partial \Omega, \mathbb{R}^{m}\right) & \rightarrow L^{p}\left(\partial \Omega, \mathbb{R}^{m}\right), \\
\mathcal{S}_{\varepsilon}: L^{p}\left(\partial \Omega, \mathbb{R}^{m}\right) & \rightarrow W^{1, p}\left(\partial \Omega, \mathbb{R}^{m}\right)
\end{aligned}
$$

are invertible and the operator norms of their inverses are bounded by a constant independent of $\varepsilon$.

Proof. The case $p=2$ was proved in [23] for Lipschitz domains. If $\Omega$ is $C^{1, \alpha}$, the results for $p \neq 2$ follow from the solvabilities of the $L^{p}$ Neumann and regularity problems with uniform estimates in $\Omega$ and $\Omega_{-}$(see Theorem 1.3 , Theorem 10.1 , Remarks 9.4 and 10.3).

As a corollary, solutions to the $L^{p}$ Dirichlet, Neumann, and regularity problems for $\mathcal{L}_{\varepsilon}\left(u_{\varepsilon}\right)=0$ may be represented by layer potentials with uniformly $L^{p}$ bounded density functions. This shows that the classical method of integral equations applies to the elliptic system $\mathcal{L}_{\varepsilon}\left(u_{\varepsilon}\right)=0$.

Theorem 11.2. Let $1<p<\infty$. Under the same assumptions on $A$ and $\Omega$ as in Theorem 11.1, the following holds.

(i) For $g \in L^{p}(\partial \Omega)$, the solution to the $L^{p}$ Dirichlet problem in $\Omega$ with $u_{\varepsilon}=g$ on $\partial \Omega$ is given by $u_{\varepsilon}=\mathcal{D}_{\varepsilon}\left(h_{\varepsilon}\right)$ with $\left\|h_{\varepsilon}\right\|_{L^{p}(\partial \Omega)} \leq C_{p}\|g\|_{L^{p}(\partial \Omega)}$.

(ii) For $g \in L^{p}(\partial \Omega)$, the solution to the $L^{p}$ Neumann problem in $\Omega$ with $\frac{\partial u_{\varepsilon}}{\partial \nu_{\varepsilon}}=g$ on $\partial \Omega$ is given by $u_{\varepsilon}=\mathcal{S}_{\varepsilon}\left(h_{\varepsilon}\right)$ with $\left\|h_{\varepsilon}\right\|_{L^{p}(\partial \Omega)} \leq C_{p}\|g\|_{L^{p}(\partial \Omega)}$.

(iii) For $g \in W^{1, p}(\partial \Omega)$, the solution to the $L^{p}$ regularity problem in $\Omega$ with $u_{\varepsilon}=g$ on $\partial \Omega$ is given by $u_{\varepsilon}=\mathcal{S}_{\varepsilon}\left(h_{\varepsilon}\right)$ with $\left\|h_{\varepsilon}\right\|_{L^{p}(\partial \Omega)} \leq C_{p}\|g\|_{L^{p}(\partial \Omega)}$.

\section{References}

[1] S. Agmon, A. Douglis, and L. Nirenberg, Estimates near the boundary for solutions of elliptic partial differential equations satisfying general boundary conditions. I, Comm. Pure Appl. Math. 12 (1959), 623-727. MR0125307 (23 \#A2610)

[2] S. Agmon, A. Douglis, and L. Nirenberg, Estimates near the boundary for solutions of elliptic partial differential equations satisfying general boundary conditions. II, Comm. Pure Appl. Math. 17 (1964), 35-92. MR0162050 (28 \#5252)

[3] Marco Avellaneda and Fang-Hua Lin, Compactness methods in the theory of homogenization, Comm. Pure Appl. Math. 40 (1987), no. 6, 803-847, DOI 10.1002/cpa.3160400607. MR.910954(88i:35019)

[4] Marco Avellaneda and Fang-Hua Lin, Homogenization of elliptic problems with L $^{p}$ boundary data, Appl. Math. Optim. 15 (1987), no. 2, 93-107, DOI 10.1007/BF01442648. MR868901 (88m:35014)

[5] Marco Avellaneda and Fang-Hua Lin, Compactness methods in the theory of homogenization. II. Equations in nondivergence form, Comm. Pure Appl. Math. 42 (1989), no. 2, 139-172, DOI 10.1002/cpa.3160420203. MR978702 (90c:35035)

[6] Marco Avellaneda and Fang-Hua Lin, Homogenization of Poisson's kernel and applications to boundary control, J. Math. Pures Appl. (9) 68 (1989), no. 1, 1-29. MR985952 (90g:35016) 
[7] M. Avellaneda and Fang-Hua Lin, $L^{p}$ bounds on singular integrals in homogenization, Comm. Pure Appl. Math. 44 (1991), no. 8-9, 897-910, DOI 10.1002/cpa.3160440805. MR1127038 (92j:42015)

[8] Alain Bensoussan, Jacques-Louis Lions, and George Papanicolaou, Asymptotic analysis for periodic structures, Studies in Mathematics and its Applications, vol. 5, North-Holland Publishing Co., Amsterdam, 1978. MR503330 (82h:35001)

[9] L. A. Caffarelli and I. Peral, On $W^{1, p}$ estimates for elliptic equations in divergence form, Comm. Pure Appl. Math. 51 (1998), no. 1, 1-21, DOI 10.1002/(SICI)10970312(199801)51:1<1::AID-CPA1>3.3.CO;2-N. MR1486629 (99c:35053)

[10] G. A. Chechkin, A. L. Piatnitski, and A. S. Shamaev, Homogenization, Translations of Mathematical Monographs, vol. 234, American Mathematical Society, Providence, RI, 2007. Methods and applications; Translated from the 2007 Russian original by Tamara Rozhkovskaya. MR2337848(2008j:35013)

[11] B. Dahlberg, personal communication (1990).

[12] Björn E. J. Dahlberg and Carlos E. Kenig, Hardy spaces and the Neumann problem in $L^{p}$ for Laplace's equation in Lipschitz domains, Ann. of Math. (2) 125 (1987), no. 3, 437-465, DOI 10.2307/1971407. MR890159 (88d:35044)

[13] A. F. M. ter Elst, Derek W. Robinson, and Adam Sikora, On second-order periodic elliptic operators in divergence form, Math. Z. 238 (2001), no. 3, 569-637, DOI 10.1007/s002090100268. MR.1869699(2003b:35087)

[14] C. Fefferman and E. M. Stein, $H^{p}$ spaces of several variables, Acta Math. 129 (1972), no. 3-4, 137-193. MR0447953 (56 \#6263)

[15] Jun Geng, $W^{1, p}$ estimates for elliptic problems with Neumann boundary conditions in Lipschitz domains, Adv. Math. 229 (2012), no. 4, 2427-2448, DOI 10.1016/j.aim.2012.01.004. MR2880228

[16] Mariano Giaquinta, Multiple integrals in the calculus of variations and nonlinear elliptic systems, Annals of Mathematics Studies, vol. 105, Princeton University Press, Princeton, NJ, 1983. MR717034 (86b:49003)

[17] Steve Hofmann and Seick Kim, The Green function estimates for strongly elliptic systems of second order, Manuscripta Math. 124 (2007), no. 2, 139-172, DOI 10.1007/s00229-007-01071. MR2341783 (2008k:35110)

[18] V. V. Jikov, S. M. Kozlov, and O. A. Oleı̆nik, Homogenization of differential operators and integral functionals, Springer-Verlag, Berlin, 1994. Translated from the Russian by G. A. Yosifian [G. A. Iosif'yan]. MR1329546 (96h:35003b)

[19] Carlos E. Kenig, Harmonic analysis techniques for second order elliptic boundary value problems, CBMS Regional Conference Series in Mathematics, vol. 83, Published for the Conference Board of the Mathematical Sciences, Washington, DC, 1994. MR.1282720 (96a:35040)

[20] C. Kenig, F. Lin, and Z. Shen, Periodic homogenization of Green and Neumann functions, Comm. Pure Appl. Math. (to appear).

[21] Carlos E. Kenig and Jill Pipher, The Neumann problem for elliptic equations with nonsmooth coefficients, Invent. Math. 113 (1993), no. 3, 447-509, DOI 10.1007/BF01244315. MR.1231834 (95b:35046)

[22] Carlos E. Kenig and Zhongwei Shen, Homogenization of elliptic boundary value problems in Lipschitz domains, Math. Ann. 350 (2011), no. 4, 867-917, DOI 10.1007/s00208-010-0586-3. MR:2818717(2012m:35039)

[23] Carlos E. Kenig and Zhongwei Shen, Layer potential methods for elliptic homogenization problems, Comm. Pure Appl. Math. 64 (2011), no. 1, 1-44, DOI 10.1002/cpa.20343. MR2743875 (2011i:35009)

[24] Joel Kilty and Zhongwei Shen, The $L^{p}$ regularity problem on Lipschitz domains, Trans. Amer. Math. Soc. 363 (2011), no. 3, 1241-1264, DOI 10.1090/S0002-9947-2010-05076-7. MR.2737264 (2012a:35072)

[25] Aekyoung Shin Kim and Zhongwei Shen, The Neumann problem in $L^{p}$ on Lipschitz and convex domains, J. Funct. Anal. 255 (2008), no. 7, 1817-1830, DOI 10.1016/j.jfa.2008.06.032. MR:2442084(2009m:35065)

[26] J.-L. Lions, Asymptotic problems in distributed systems, Metastability and incompletely posed problems (Minneapolis, Minn., 1985), IMA Vol. Math. Appl., vol. 3, Springer, New York, 1987, pp. 241-258, DOI 10.1007/978-1-4613-8704-6_14. MR870019 (87m:93076) 
[27] J.-L. Lions, Exact controllability, stabilization and perturbations for distributed systems, SIAM Rev. 30 (1988), no. 1, 1-68, DOI 10.1137/1030001. MR 931277(89e:93019)

[28] O. A. Oleĭnik, A. S. Shamaev, and G. A. Yosifian, Mathematical problems in elasticity and homogenization, Studies in Mathematics and its Applications, vol. 26, North-Holland Publishing Co., Amsterdam, 1992. MR1195131 (93k:35025)

[29] Zhongwei Shen, Bounds of Riesz transforms on $L^{p}$ spaces for second order elliptic operators, Ann. Inst. Fourier (Grenoble) 55 (2005), no. 1, 173-197 (English, with English and French summaries). MR2141694(2006a:35045)

[30] Zhongwei Shen, Necessary and sufficient conditions for the solvability of the $L^{p}$ Dirichlet problem on Lipschitz domains, Math. Ann. 336 (2006), no. 3, 697-725, DOI 10.1007/s00208006-0022-x. MR2249765(2008e:35059)

[31] Zhongwei Shen, The $L^{p}$ boundary value problems on Lipschitz domains, Adv. Math. 216 (2007), no. 1, 212-254, DOI 10.1016/j.aim.2007.05.017. MR2353255 (2009a:35064)

[32] Michael E. Taylor, Tools for PDE, Mathematical Surveys and Monographs, vol. 81, American Mathematical Society, Providence, RI, 2000. Pseudodifferential operators, paradifferential operators, and layer potentials. MR 1766415 (2001g:35004)

[33] Gregory Verchota, Layer potentials and regularity for the Dirichlet problem for Laplace's equation in Lipschitz domains, J. Funct. Anal. 59 (1984), no. 3, 572-611, DOI 10.1016/00221236(84)90066-1. MR769382 (86e:35038)

Department of Mathematics, University of Chicago, Chicago, Illinois 60637

E-mail address: cek@math.uchicago.edu

Courant Institute of Mathematical Sciences, New York University, New York, New YORK 10012

E-mail address: linf@cims.nyu.edu

Department of Mathematics, University of Kentucky, Lexington, Kentucky 40506

E-mail address: zshen2@email.uky.edu 